\title{
MULTIPLIER THEOREMS VIA MARTINGALE TRANSFORMS
}

\author{
RODRIGO BAÑUELOS, FABRICE BAUDOIN, LI CHEN, AND YANNICK SIRE
}

\begin{abstract}
We develop a new and general approach to prove multiplier theorems in various geometric settings. The main idea is to use martingale transforms and a Gundy-Varopoulos representation for multipliers defined via a suitable extension procedure. Along the way, we provide a probabilistic proof of a generalization of a result by Stinga and Torrea, which is of independent interest. Our methods here also recover the sharp $L^{p}$ bounds for second order Riesz transforms by a liming argument.
\end{abstract}

\section{Contents}

1. Introduction and main results

2. Preliminaries, Extension procedure

2.1. Setting

2.2. Green function at $+\infty$ of one-dimensional diffusions killed at 0

2.3. Extension procedure with general vertical diffusions

2.4. Martingale inequalities

3. Multiplier theorems

3.1. Construction of the martingale transform associated to a multiplier

3.2. Boundedness in $L^{p}$

3.3. Specific choices for the vertical diffusion 14

4. Generalized Riesz transforms 16

4.1. Operators arising from martingale transforms 17

4.2. $\begin{array}{ll}\text { Boundedness in } L^{p}(\mu) & 18\end{array}$

4.3. Euclidean spaces and Lie groups of compact type 19

4.4. Generalized Riesz transform on vector bundles 25

Acknowledgements $\quad 26$

$\begin{array}{ll}\text { References } & 26\end{array}$

\section{Introduction AND MAIN RESUlts}

The $L^{p}$-boundedness properties of Riesz transforms in wide geometric settings have been extensively studied by a large number of authors for many years. The large literature on this topic includes techniques from the Calderón-Zygmund theory of singular integrals and probabilistic and analytic Littlewood-Paley theory. For some of this literature we refer the reader to [4], [14] and [8]. On the other hand,

Date: July 13, 2021.

R. Bañuelos supported in part by NSF Grant DMS-1854709. F. Baudoin supported in part by NSF Grant DMS-1901315. 
the probabilistic approach of R. F. Gundy and N. Th. Varopoulos [28] which represents the Riesz transforms as conditional expectations of martingale transforms, combined with the sharp martingale inequalities of D.L. Burkholder, provides a powerful tool to obtain not only $L^{p}$ bounds with constant that do not depend on the geometry of the ambient space but often give sharp, or nearly sharp, bounds. The martingale techniques also apply to Riesz transforms on Wiener space providing explicit bounds. For an incomplete list of references to this now very large literature, we refer to [8] and [12]. In addition to providing universal and explicit $L^{p}$ bounds, the martingale transform techniques extend to multipliers beyond Riesz transforms. For some of this literature, we refer to [6]. A common thread in the Gundy-Varopoulos constructions has been to build the martingale transforms on stochastic processes of the form $\left(X_{t}, Y_{t}\right)$ where $X_{t}$ is either a diffusion or a process arising from a Markovian semigroup on $\mathbb{R}^{n}$ or on a manifold $M$ (such as the Lévy multipliers studied in [9]), and where $Y_{t}$ is either a one dimensional Brownian motion on $\mathbb{R}^{+}$killed upon hitting 0 (harmonic extensions) or $T-t$ for some fixed time $T$, in the case of space-time (heat extension) constructions as in [7]. The goal of this paper is to prove boundedness of multipliers obtained when the "vertical" process $Y_{t}$ is more general than those just mentioned. More precisely, we will study multipliers that arise as conditional expectations of martingale transforms which are built on the process $\left(X_{t}, \eta_{t}\right)$ where the vertical diffusion has a generator of the form given in (5). As we show in Section 4 (see Remark 4.7), our construction unifies both the original constructions with $\left(X_{t}, Y_{t}\right)$ of Gundy-Varopoulos, which gives sharp inequalities for first order Riesz transforms [13], and the construction for $\left(X_{t}, T-t\right)$ from [5], which gives sharp inequalities for second order Riesz transforms, into one by a limiting procedure.

The last two decades or so have seen a great amount of works dealing with nonlocal operators (generators of Lévy processes) from the PDE point of view (see e.g. the recent book [36]). In particular, the paper [20] has been instrumental in interpreting fractional powers of the Laplacian in $\mathbb{R}^{n}$ in terms of a suitable "harmonic" extension. Note that in the language of probability, this result had been proved in [35]. This latter result has been put in a more general (and flexible) framework by Stinga and Torrea in [40]. It is beyond the scope of this paper to review the amount of works using such technique. Our contributions here lie at the interface of probabilistic methods and harmonic analysis. More precisely, in the present paper, combining the Gundy-Varopoulos approach to Riesz transforms and a probabilistic approach to the result of Stinga and Torrea, we obtain new results about the boundedness in $L^{p}$ of three types of operators:

- Multipliers of the type $\Phi(-\Delta+V)$, where $\Delta$ is a diffusion operator and $V$ a non-negative bounded smooth potential;

- Generalized Riesz transforms of the type $\Phi(-\Delta+V) \mathfrak{X}_{i}$, where the $\mathfrak{X}_{i}$ 's are first-order differential operators that commute with $-\Delta+V$;

- Generalized second order Riesz transforms of the type $\Phi(-\Delta+V) \mathfrak{X}_{i} \mathfrak{X}_{j}$.

We note that using methods from harmonic analysis many results about $L^{p}$ estimates for Schrödinger operators are already available in the literature in some settings and various more general assumptions on the potential $V$, see for instance $[2,39]$. However, those methods rely heavily on the geometry of the underlying space and yield dimension dependent $L^{p}$-bounds. The probabilistic method we are using here uses stronger assumptions on the potential $V$ but on the other hand 
yields dimension independent $L^{p}$-bounds and relies very little on the geometry of the underlying space.

When $V=0$ among other things, we prove the following general multiplier theorem. Let $\Delta$ be a locally subelliptic (in the sense of Fefferman-Phong) diffusion operator on a smooth manifold $M$ which is essentially self-adjoint on the space of smooth and compactly supported functions with respect to a measure $\mu$ on $M$. We assume that $\Delta$ generates a diffusion process $\left(\left(X_{t}\right)_{t>0},\left(\mathbb{P}_{x}\right)_{x \in M}\right)$ which is not explosive. If $\Phi$ is a bounded Borel function on $[0,+\infty)$ the operator $\Phi(-\Delta)$ may be defined on $L^{2}(M, \mu)$ by using the spectral theorem. By using martingale transforms, we will then prove the following theorem.

Theorem 1.1. If there exists a finite complex Borel measure $\alpha$ on $\mathbb{R}_{\geq 0}$ such that for every $x \in[0,+\infty)$,

$$
\Phi(x)=\int_{0}^{+\infty}\left(1-\frac{m}{\sqrt{m^{2}+x}}\right) d \alpha(m),
$$

then, for every $p>1$ and $f \in L^{p}(M, \mu)$,

$$
\|\Phi(-\Delta) f\|_{p} \leq 2\left(p^{*}-1\right)|\alpha|([0,+\infty))\|f\|_{p}
$$

where $p^{*}=\max \left\{p, \frac{p}{p-1}\right\}$.

In Theorem 3.6 below, we actually prove a more general result that also applies to Schrödinger operators. The representation (1) is related to the theory of Stieltjes transforms, see $[29,44]$, and is possible to invert. We note that Theorem 1.1 can also be proved using Bernstein theorem, since the function $x \rightarrow \frac{m}{\sqrt{m^{2}+x}}$ is completely monotone. However, the method we propose is general and is easily adapted to study different multipliers as generalized first order or second order Riesz transforms.

Concerning the study of generalized first order and second order Riesz transforms on Lie groups of compact type, using a variation of the method to construct multipliers, we obtain the following result.

Theorem 1.2. Let $G$ be a n-dimensional Lie group of compact type endowed with a bi-invariant Riemannian structure. Let $\mathfrak{X}_{1}, \cdots, \mathfrak{X}_{n}$ be an orthonormal frame of the Lie algebra of $G$ and denote by $\Delta$ the Laplace Beltrami operator on $G$. Let $\Phi:(0,+\infty) \rightarrow \mathbb{C}$ be a complex Borel function.

(1) If there exists a finite complex Borel measure $\alpha$ on $[0,+\infty)$ such that for every $x \in(0,+\infty)$,

$$
\Phi(x)=\int_{0}^{+\infty} \frac{d \alpha(m)}{\sqrt{x+m}}
$$

then, for every $1 \leq i \leq n, p>1$, and $f \in L^{p}$

$$
\left\|\Phi(-\Delta) \mathfrak{X}_{i} f\right\|_{p} \leq \cot \left(\frac{\pi}{2 p^{*}}\right)|\alpha|([0,+\infty))\|f\|_{p} .
$$

(2) If there exists a finite complex Borel measure $\alpha$ on $[0,+\infty]$ such that for every $x \in(0,+\infty)$

$$
\Phi(x)=\int_{0}^{+\infty} \frac{d \alpha(m)}{\sqrt{x+m^{2}}\left(\sqrt{x+m^{2}}-m\right)},
$$


then, for every $1 \leq i, j \leq d, p>1$, and $f \in L^{p}$

$$
\left\|\Phi(-\Delta) \frac{1}{2}\left(\mathfrak{X}_{i} \mathfrak{X}_{j}+\mathfrak{X}_{j} \mathfrak{X}_{i}\right) f\right\|_{p} \leq\left(p^{*}-1\right)|\alpha|([0,+\infty])\|f\|_{p} .
$$

Theorem 1.2 is sharp. Indeed, in (2), if one choses $\alpha$ to be the Dirac distribution at 0 , one gets

$$
\left\|(-\Delta)^{-1 / 2} \mathfrak{X}_{i} f\right\|_{p} \leq \cot \left(\frac{\pi}{2 p^{*}}\right)\|f\|_{p}
$$

which is the sharp bound for the Riesz transform, see [13] and [31]. In (3), if one choses $\alpha$ to be the Dirac distribution at $+\infty$, one gets

$$
\left\|(-\Delta)^{-1} \frac{1}{2}\left(\mathfrak{X}_{i} \mathfrak{X}_{j}+\mathfrak{X}_{j} \mathfrak{X}_{i}\right) f\right\|_{p} \leq \frac{1}{2}\left(p^{*}-1\right)\|f\|_{p}
$$

which is the sharp bound for the second order Riesz transform, see [5] and [24].

Finally, using techniques developed in [8] to handle the study of Riesz transforms on vector bundles, we obtain the following result.

Theorem 1.3. Let $M$ be a complete Riemannian manifold with non-negative Weitzenböck curvature. Let $\mathcal{L}=d d^{*}+d^{*} d$ be the Hodge-de Rham Laplace operator on the exterior bundle of $M$. Let $\Phi:(0,+\infty) \rightarrow \mathbb{C}$ be a complex Borel function. If there exists a finite complex Borel measure $\alpha$ on $\mathbb{R}_{\geq 0}$ such that for every $x \in(0,+\infty)$,

$$
\Phi(x)=\int_{0}^{+\infty} \frac{d \alpha(m)}{\sqrt{x+m}}
$$

then, for every $p>1$ and every $L^{p}$ integrable exterior differential form $\eta$

$$
\|\Phi(\mathcal{L}) d \eta\|_{p} \leq 6\left(p^{*}-1\right)|\alpha|([0,+\infty))\|\eta\|_{p} .
$$

\section{Preliminaries, Extension procedure}

2.1. Setting. Let $\Delta$ be a locally subelliptic diffusion operator (see Section 1.2 in [18] and [32] for a definition of local subellipticity) on a smooth manifold $M$. For every smooth functions $f, g: M \rightarrow \mathbb{R}$, we define the so-called carré du champ operator, which is the symmetric first-order differential form defined by:

$$
\Gamma(f, g)=\frac{1}{2}(\Delta(f g)-f \Delta g-g \Delta f) .
$$

A straightforward computation shows that in a local chart one has

$$
\Delta=\sum_{i, j=1}^{n} \sigma_{i j}(x) \frac{\partial^{2}}{\partial x_{i} \partial x_{j}}+\sum_{i=1}^{n} b_{i}(x) \frac{\partial}{\partial x_{i}}
$$

where $\left(\sigma_{i j}(x)\right)$ is symmetric and nonnegative. That is, for $1 \leq i, j \leq n, \sigma_{i j}(x)=$ $\sigma_{j i}(x)$ and for $\xi \in \mathbb{R}^{n}, \sum_{i, j=1}^{n} \sigma_{i j}(x) \xi_{i} \xi_{j} \geq 0$. Then in the same chart

$$
\Gamma(f, g)=\sum_{i, j=1}^{n} \sigma_{i j}(x) \frac{\partial f}{\partial x_{i}} \frac{\partial g}{\partial x_{j}} .
$$


As a consequence, for every smooth function $f, \Gamma(f):=\Gamma(f, f) \geq 0$. We assume that $\Delta$ is symmetric with respect to some smooth measure $\mu$, which means that for every smooth and compactly supported functions $f, g \in C_{0}^{\infty}(M)$,

$$
\int_{M} g \Delta f d \mu=\int_{M} f \Delta g d \mu \text {. }
$$

By smooth measure $\mu$, we mean here that $\mu$ is given by a density in the sense of Definition 3.90 in [23]. That is, locally, in any coordinate system $x_{i}, \mu$ has a smooth density with respect to the volume form $\left|d x_{1} \wedge \cdots \wedge d x_{n}\right|$. There is an intrinsic distance associated to the operator $\Delta$ which is defined by

$$
d(x, y)=\sup \left\{|f(x)-f(y)|, f \in C^{\infty}(M),\|\Gamma(f)\|_{\infty} \leq 1\right\}, \quad x, y \in M .
$$

We assume that the metric space $(M, d)$ is complete. In that case, from Propositions 1.20 and 1.21 in [18], the operator $\Delta$ is essentially self-adjoint on $C_{0}^{\infty}(M)$.

Let now $V: M \rightarrow \mathbb{R}$ be a non-positive lower bounded smooth potential and consider the Schrödinger operator

$$
L=\Delta+V .
$$

The operator $L$ is also essentially self-adjoint on the space of smooth and compactly supported functions. Indeed from Proposition 1.21 in [18], there exists a sequence $h_{n} \in C_{0}^{\infty}(M), 0 \leq h_{n} \leq 1$ such that $h_{n} \nearrow 1$ and $\left\|\Gamma\left(h_{n}\right)\right\|_{\infty} \rightarrow 0$. Using then the argument in the proof of Proposition 1.20 in [18] together with the fact that $V \leq 0$ yields the fact that $L$ is essentially self-adjoint on $C_{0}^{\infty}(M)$. The self-adjoint extension of $L$ will still be denoted by $L$. The semigroup in $L^{2}(M, \mu)$ generated by $L$ will be denoted by $\left(P_{t}\right)_{t \geq 0}$.

We assume that $\Delta$ generates a diffusion process $\left(X_{t},\left(\mathbb{P}_{x}\right)_{x \in M}\right)$ which is not explosive. In that case, the Schrödinger semigroup $\left(P_{t}\right)_{t>0}$ admits the FeynmanKac representation (see for instance [17, Theorem 6.20]):

$$
P_{t} f(x)=\mathbb{E}^{x}\left(e^{\int_{0}^{t} V\left(X_{s}\right) d s} f\left(X_{t}\right)\right), \quad f \in C_{0}^{\infty}(M) .
$$

The semigroup $\left(P_{t}\right)_{t \geq 0}$ hence defined is then a sub-Markov semigroup (see page 71 in [17] for a definition and basic properties of sub-Markov semigroups).

Remark 2.1. It is a well-known result by A. Grigor'yan [27, Theorem 1] and K.T. Sturm [41, Theorem 4] that a sufficient condition for $\Delta$ to generates a diffusion process $\left(X_{t},\left(\mathbb{P}_{x}\right)_{x \in M}\right)$ which is not explosive is that for some $x_{0} \in M$ and $r_{0}>0$

$$
\int_{r_{0}}^{+\infty} \frac{r d r}{\ln \mu\left(B\left(x_{0}, r\right)\right)}=+\infty
$$

where $B\left(x_{0}, r\right)$ denotes the metric ball with radius $r$ for the distance $d$. This is for instance satisfied if for some constants $C_{1}, C_{2}>0$ one has $\mu\left(B\left(x_{0}, r\right)\right) \leq C_{1} e^{C_{2} r^{2}}$.

2.2. Green function at $+\infty$ of one-dimensional diffusions killed at $\mathbf{0}$. Let $a, b$ be smooth functions on $(0, \infty)$ with $a>0$ and let

$$
s^{\prime}(z)=\exp \left(-\int_{1}^{z} \frac{b(y)}{a(y)^{2}} d y\right) .
$$

Assume that

$$
\int_{1}^{\infty} s^{\prime}(z) d z=\infty, \quad \int_{0}^{1} s^{\prime}(z) d z<\infty .
$$


We consider a one-dimensional diffusion operator on $(0,+\infty)$

$$
\mathcal{B}=a(y)^{2} \frac{\partial^{2}}{\partial y^{2}}+b(y) \frac{\partial}{\partial y}
$$

with Dirichlet boundary condition at 0 . The quantities $s^{\prime}(z)$ and $m(z):=\frac{1}{s^{\prime}(z) a(z)^{2}}$ are respectively often called the scale function and density of the speed measure associated with the diffusion $\mathcal{B}$. For more on this, see [19, Section II.9] or [38, Chapter VII, Definitions 3.3 and 3.7].

Let $\eta_{t}$ be the diffusion process with generator $\mathcal{B}$. We denote

$$
\tau=\inf \left\{t>0, \eta_{t}=0\right\} .
$$

and $q_{t}(y)$ the density of $\tau$ under $\mathbb{P}_{y}, \eta_{0}=y>0$. It is well known that under the assumption (4), the process $\eta$ is not explosive and hits zero with probability 1 (see for instance [38, Ch VII Proposition 3.2]), that is,

$$
\mathbb{P}(\tau<+\infty)=1 \text {. }
$$

For later use, we assume that $\eta$ can be written as a (weak) solution of a SDE

$$
d \eta_{t}=b\left(\eta_{t}\right) d t+a\left(\eta_{t}\right) d \beta_{t}, \quad t<\tau
$$

where $\beta_{t}$ is a Brownian motion on $\mathbb{R}$ with $\mathbb{E}\left(\beta_{t}^{2}\right)=2 t$, which is independent of the process $\left(X_{t}\right)_{t \geq 0}$. We first collect some preliminary results about the Green function at $+\infty$ of the diffusion $\eta$ killed at 0 . For computations, it is convenient to write $\mathcal{B}$ as

$$
\mathcal{B}=a(y)^{2} \frac{\partial^{2}}{\partial y^{2}}+a(y)^{2} \frac{h^{\prime}(y)}{h(y)} \frac{\partial}{\partial y},
$$

where $h$ is a nonnegative function such that $a(y)^{2} \frac{h^{\prime}(y)}{h(y)}=b(y)$. Note that one can choose

$$
h(y)=\exp \left(\int_{1}^{y} \frac{b(w)}{a(w)^{2}} d w\right)
$$

so that the assumptions in (4) imply

$$
\int_{0}^{1} \frac{d w}{h(w)}<+\infty, \quad \int_{1}^{+\infty} \frac{d w}{h(w)}=+\infty
$$

The following lemma which computes the Green function of $\mathcal{B}$ on the half-line $[0,+\infty)$ with Dirichlet boundary condition at 0 is then straightforward.

Lemma 2.2. Let $g$ be a Borel function such that $\int_{0}^{+\infty} h(z) \frac{|g(z)|}{a(z)^{2}} d z<+\infty$. The solution on $[0,+\infty)$ of the equation

$$
\mathcal{B} f=-g
$$

with boundary conditions $f(0)=0$ and $\left(f^{\prime} h\right)(+\infty)=0$, is given by

$$
f(y)=\int_{0}^{+\infty} G(y, z) g(z) d z
$$

where

In particular,

$$
G(y, z)=\frac{h(z)}{a(z)^{2}} \int_{0}^{z \wedge y} \frac{d w}{h(w)}
$$

$$
G(+\infty, z):=\lim _{y \rightarrow+\infty} G(y, z)=\frac{h(z)}{a(z)^{2}} \int_{0}^{z} \frac{d w}{h(w)}=s(z) m(z) .
$$


Proof. Notice that the equation $\mathcal{B} f=-g$ can be rewritten as

$$
a(y)^{2} f^{\prime \prime}(y)+a(y)^{2} \frac{h^{\prime}(y)}{h(y)} f^{\prime}(y)=-g(y),
$$

where $h(y)=\exp \left(\int_{1}^{y} \frac{b(w)}{a(w)^{2}} d w\right)$. This is equivalent to

$$
\frac{1}{h(y)}\left(f^{\prime} h\right)^{\prime}(y)=-\frac{g(y)}{a(y)^{2}} .
$$

Since $\int_{0}^{+\infty} h(z) \frac{|g(z)|}{a(z)^{2}} d z<+\infty$, the first order ODE with boundary condition $\left(f^{\prime} h\right)(+\infty)=$ 0 has a unique solution

$$
f^{\prime}(y) h(y)=\int_{y}^{+\infty} h(z) \frac{g(z)}{a(z)^{2}} d z .
$$

Again from the boundary condition $f(0)=0$, we conclude the unique existence of the solution $f$ as

$$
\begin{aligned}
f(y) & =\int_{0}^{y} \frac{1}{h(z)} \int_{z}^{+\infty} h(w) \frac{g(w)}{a(w)^{2}} d w d z \\
& =\int_{0}^{+\infty} \int_{0}^{w \wedge y} \frac{d z}{h(z)} \frac{h(w)}{a(w)^{2}} g(w) d w .
\end{aligned}
$$

Our next lemma is the occupation time formula for the process $\eta_{t}$.

Lemma 2.3. Let $G$ be the Green function of $\mathcal{B}$ on the half-line $[0,+\infty)$ with Dirichlet boundary condition at 0 as above. Then, if $g$ is a positive Borel function such that $\int_{0}^{+\infty} h(z) \frac{g(z)}{a(z)^{2}} d z<+\infty$, for every $y>0$,

$$
\mathbb{E}_{y}\left(\int_{0}^{\tau} g\left(\eta_{s}\right) d s\right)=f(y)
$$

where $f$ solves the equation in Lemma 2.2.

Proof. Let $f$ be the solution of

$$
\mathcal{B} f=-g
$$

with boundary conditions $f(0)=0$ and $\left(f^{\prime} h\right)(+\infty)=0$. By Itô's formula,

$$
f\left(\eta_{t}\right)=f\left(\eta_{0}\right)+\int_{0}^{t} f^{\prime}\left(\eta_{s}\right) a\left(\eta_{s}\right) d \beta_{s}+\int_{0}^{t} \mathcal{B} f\left(\eta_{s}\right) d s, \quad t<\tau
$$

In particular, letting $t \rightarrow \tau$, one obtains

$$
f\left(\eta_{0}\right)=\int_{0}^{\tau} g\left(\eta_{s}\right) d s-\int_{0}^{\tau} f^{\prime}\left(\eta_{s}\right) a\left(\eta_{s}\right) d \beta_{s} .
$$

Denote by $\tau_{n}=\tau \wedge \sigma_{n} \wedge n$, where $\sigma_{n}=\inf \left\{t \geq 0, \eta_{t}=n\right\}$. Since $a$ and $f^{\prime}$ are smooth in $(0, \infty)$ and $\tau_{n}$ is a bounded stopping time, $\left(\int_{0}^{t \wedge \tau_{n}} f^{\prime}\left(\eta_{s}\right) a\left(\eta_{s}\right) d \beta_{s}\right)_{t \geq 0}$, is a martingale. Applying the Doob's optional stopping theorem we get

$$
\mathbb{E}^{y}\left(\int_{0}^{\tau_{n}} f^{\prime}\left(\eta_{s}\right) a\left(\eta_{s}\right) d \beta_{s}\right)=\mathbb{E}^{y}\left(f\left(\eta_{\tau_{n}}\right)-f\left(\eta_{0}\right)-\int_{0}^{\tau_{n}} \mathcal{B} f\left(\eta_{s}\right) d s\right)=0 .
$$


This gives

$$
f(y)=\mathbb{E}^{y}\left(\int_{0}^{\tau_{n}} g\left(\eta_{s}\right) d s\right)+\mathbb{E}^{y}\left(f\left(\eta_{\tau_{n}}\right)\right) .
$$

Letting $n \rightarrow \infty$, the monotone convergence theorem yields

$$
f(y)=\mathbb{E}^{y}\left(\int_{0}^{\tau} g\left(\eta_{s}\right) d s\right) .
$$

2.3. Extension procedure with general vertical diffusions. If $f \in L^{2}(M, \mu)$ we consider its extension to the cone $M \times[0,+\infty)$ defined for $x \in M, y \in[0,+\infty)$ by

$$
U_{f}(x, y)=\int_{0}^{+\infty} P_{t} f(x) q_{t}(y) d t,
$$

where we recall that $P_{t}=e^{t L}$ is the semigroup generated by $L=\Delta+V$ and that $q_{t}$ is the density of the first hitting time $\tau$ of zero by $\eta$. Since $\Delta$ is locally subelliptic and $V$ is smooth, using the definition of local subellipticity for $\Delta$ (see Definitions 1.6 and 1.8 in [18] ) one deduces that $L$ is itself locally subelliptic. Therefore $P_{t} f$ is a smooth function for every $f \in L^{2}(M, \mu)$ (see Proposition 1.23 in [18]).

Denoting

$$
\mathcal{K}(y, \lambda):=\int_{0}^{+\infty} e^{-\lambda t} q_{t}(y) d t=\mathbb{E}^{y}\left(e^{-\lambda \tau}\right),
$$

we have that $\mathcal{B K}(\cdot, \lambda)=\lambda \mathcal{K}(\cdot, \lambda)$. In particular, since $\mathcal{B}$ is an elliptic operator, we deduce that $\mathcal{K}(\cdot, \lambda)$ is a smooth function (see page 18 in [19] for more details, including a representation formula for $\mathcal{K}(\cdot, \lambda))$.

We note that the spectral theorem shows that in the $L^{2}$ sense

$$
U_{f}(x, y)=\mathcal{K}(y,-L) f(x) .
$$

The starting point of our approach is the following generalization of a result by Stinga and Torrea (see [40]).

Theorem 2.4. Let $f \in C_{0}^{\infty}(M)$. In the pointwise sense $U_{f}$ satisfies

$$
\begin{cases}(L+\mathcal{B}) U_{f}=0 & \text { in } M \times(0,+\infty) \\ U(\cdot, 0)=f & \text { on } M .\end{cases}
$$

We shall give a probabilistic proof of this result which is based on a martingale that shall be used several times in this paper.

Lemma 2.5. Let $f \in C_{0}^{\infty}(M)$. Consider the process

$$
M_{t}^{f}=e^{\int_{0}^{t \wedge \tau} V\left(X_{u}\right) d u} U_{f}\left(X_{t \wedge \tau}, \eta_{t \wedge \tau}\right) .
$$

The process $M_{t}^{f}$ is a martingale with quadratic variation

$\left\langle M^{f}\right\rangle_{t}=2 \int_{0}^{t \wedge \tau} e^{2 \int_{0}^{s} V\left(X_{u}\right) d u} \Gamma\left(U_{f}\right)\left(X_{s}, \eta_{s}\right) d s+2 \int_{0}^{t \wedge \tau} e^{2 \int_{0}^{s} V\left(X_{u}\right) d u} \partial_{y} U_{f}\left(X_{s}, \eta_{s}\right)^{2} a\left(\eta_{s}\right)^{2} d s$,

where $\Gamma\left(U_{f}\right)=\sum_{i, j=1}^{n} \sigma_{i j} \frac{\partial U_{f}}{\partial x_{i}} \frac{\partial U_{f}}{\partial x_{j}}$. 
Proof. First note that

$$
M_{\tau}^{f}=e^{\int_{0}^{\tau} V\left(X_{u}\right) d u} f\left(X_{\tau}\right) .
$$

Assume that the process $\left(X_{t}, \eta_{t}\right)_{t \geq 0}$ starts at $(x, y) \in M \times(0, \infty)$. Since the processes $X_{t}$ and $\eta_{t}$ are independent, it follows from the Feynman-Kac formula that

$$
\begin{aligned}
\mathbb{E}^{x, y}\left(e^{\int_{0}^{\tau} V\left(X_{u}\right) d u} f\left(X_{\tau}\right)\right) & =\int_{0}^{\infty} \mathbb{E}^{x}\left(e^{\int_{0}^{s} V\left(X_{u}\right) d u} f\left(X_{s}\right)\right) q_{s}(y) d s \\
& =\int_{0}^{\infty} P_{s} f(x) q_{s}(y) d s=U_{f}(x, y),
\end{aligned}
$$

where we recall that $q_{s}(y)$ is the density of $\tau$ under $\eta_{0}=y>0$. Denote by $\left(\mathcal{F}_{t}\right)_{t \geq 0}$ the natural filtration of $\left(X_{t}, \eta_{t}\right)$. From the strong Markov property we have

$$
\begin{aligned}
\mathbb{E}\left(M_{\tau}^{f} \mid \mathcal{F}_{s \wedge \tau}\right) & =\mathbb{E}\left(e^{\int_{0}^{\tau} V\left(X_{u}\right) d u} f\left(X_{\tau}\right) 1_{\tau \leq s} \mid \mathcal{F}_{s \wedge \tau}\right)+\mathbb{E}\left(e^{\int_{0}^{\tau} V\left(X_{u}\right) d u} f\left(X_{\tau}\right) 1_{\tau>s} \mid \mathcal{F}_{s \wedge \tau}\right) \\
& =e^{\int_{0}^{\tau} V\left(X_{u}\right) d u} f\left(X_{\tau}\right) 1_{\tau \leq s}+e^{\int_{0}^{s \wedge \tau} V\left(X_{u}\right) d u} U_{f}\left(X_{s \wedge \tau}, \eta_{s \wedge \tau}\right) 1_{\tau>s} \\
& =M_{s \wedge \tau}^{f} .
\end{aligned}
$$

We conclude that $M_{t}^{f}$ is a martingale. The quadratic variation of $M_{t}^{f}$ is computed as in $[38$, p.324] or $[15$, p. 181]. Indeed, from Itô's formula, the bounded variation part of $M_{t}^{f}$ is zero. Hence

$$
\begin{array}{r}
M_{t}^{f}=U_{f}(x, y)+\sum_{i=1}^{n} \int_{0}^{t \wedge \tau} e^{\int_{0}^{s} V\left(X_{u}\right) d u}\left(\sum_{j=1}^{n} v_{i j} \partial_{x_{j}}\right) U_{f}\left(X_{s}, \eta_{s}\right) d B_{s}^{i} \\
+\int_{0}^{t \wedge \tau} e^{\int_{0}^{s} V\left(X_{u}\right) d u} \partial_{y} U_{f}\left(X_{s}, \eta_{s}\right) a\left(\eta_{s}\right) d \beta_{s},
\end{array}
$$

where $\left(v_{i j}(x)\right)$ is the square root of the symmetric nonnegative matrix $\left(\sigma_{i j}(x)\right)$ and $B_{t}=\left(B_{t}^{1}, \cdots, B_{t}^{n}\right)$ is a Brownian motion on $\mathbb{R}^{n}$ with generator $\Delta=\sum_{i=1}^{n} \frac{\partial^{2}}{\partial x_{i}^{2}}$. Thus $\left\langle M^{f}\right\rangle_{t}$ immediately follows.

We are now in position to prove Theorem 2.4.

Proof of Theorem 2.4. Since $M_{t}^{f}=e^{\int_{0}^{t \wedge \tau} V\left(X_{u}\right) d u} U_{f}\left(X_{t \wedge \tau}, \eta_{t \wedge \tau}\right)$ is a martingale, it follows from Itô's formula that the bounded variation part of $M_{t}^{f}$ is zero, i.e.,

$$
\int_{0}^{t \wedge \tau} e^{\int_{0}^{s} V\left(X_{u}\right) d u}(L+\mathcal{B}) U_{f}\left(X_{s}, \eta_{s}\right) d s=0 .
$$

We conclude that

$$
(L+\mathcal{B}) U_{f}(x, y)=\lim _{t \rightarrow 0} \frac{1}{t} \int_{0}^{t \wedge \tau} e^{\int_{0}^{s} V\left(X_{u}\right) d u}(L+\mathcal{B}) U_{f}\left(X_{s}, \eta_{s}\right) d s=0 .
$$

2.4. Martingale inequalities. In this section, we recall some results on the martingale inequalities used in this paper. Suppose that $(\Omega, \mathcal{F}, \mathbb{P})$ is a complete probability space, filtered by $\mathcal{F}=\left\{\mathcal{F}_{t}\right\}_{t \geq 0}$, a family of right continuous sub- $\sigma$-fields of $\mathcal{F}$. Assume that $\mathcal{F}_{0}$ contains all the events of probability 0 . Let $X$ and $Y$ be adapted, real-valued martingales which have right-continuous paths with left-limits (r.c.l.l.). The martingale $Y$ is differentially subordinate to $X$ if $\left|Y_{0}\right| \leq\left|X_{0}\right|$ and $\langle X\rangle_{t}-\langle Y\rangle_{t}$ 
is a nondecreasing and nonnegative function of $t$. The martingales $X_{t}$ and $Y_{t}$ are said to be orthogonal if the covariation process $\langle X, Y\rangle_{t}=0$ for all $t$.

We always assume that the martingales are $L^{p}$ bounded for $1<p<\infty$ and by $X$ we mean $X_{\infty}$. By $\|X\|_{p}$ we mean $\sup _{t>0}\left\|X_{t}\right\|_{p}=\left\|X_{\infty}\right\|_{p}$. This is often applied to stopped martingales. Hence $\|X\|_{p}=\left\|X_{\tau}\right\|_{p}$ where $\tau$ is a stopping time. We use the notation $\|f\|_{p}$ for the $L^{p}$-norms of functions defined on $M$ with respect to the measure $\mu$. This is clear in each occurrence and should not create any confusion.

In the following, we recall the sharp inequalities of martingale transforms proved by Bañuelos and Wang [13], as well as an extension by Bañuelos and Osȩkowski [12].

Theorem 2.6 ([13, Theorems 1 and 2]). Let $X$ and $Y$ be two martingales with continuous paths such that $Y$ is differentially subordinate to $X$. Fix $1<p<\infty$ and set $p^{*}=\max \left\{p, \frac{p}{p-1}\right\}$. Then

$$
\|Y\|_{p} \leq\left(p^{*}-1\right)\|X\|_{p} .
$$

Furthermore, suppose that the martingales $X$ and $Y$ are orthogonal. Then

$$
\|Y\|_{p} \leq \cot \left(\frac{\pi}{2 p^{*}}\right)\|X\|_{p}
$$

Both of these inequalities are sharp.

Theorem 2.7 ([12, Theorem 2.2]). Let $X$ and $Y$ be two martingales with continuous paths such that $Y$ is differentially subordinate to $X$. Consider the process

$$
Z_{t}=e^{\int_{0}^{t} V_{s} d s} \int_{0}^{t} e^{-\int_{0}^{s} V_{v} d v} d Y_{s}
$$

where $\left(V_{t}\right)_{t \geq 0}$ is a non-positive adapted and continuous process. For $1<p<\infty$, we have the sharp bound

$$
\|Z\|_{p} \leq\left(p^{*}-1\right)\|X\|_{p}
$$

\section{Multiplier theorems}

The martingale transform method to construct multipliers is very versatile and allows one to deal with a very general setup. We work under the assumptions and with the notations of Section 2. In particular, let $G$ be the Green function of $\mathcal{B}$ on the half-line $[0,+\infty$ ) with Dirichlet boundary condition at 0 (see Lemma 2.2). Before going further, in order to put the next results in perspective, we discuss the well-known Hörmander-Mikhlin theorem in $\mathbb{R}^{n}$. Let us assume that $\Delta=\sum_{i=1}^{n} \frac{\partial^{2}}{\partial x_{i}^{2}}$ is the Laplace operator in $\mathbb{R}^{n}$ and that $V=0$. Recall the Hörmander-Mikhlin theorem:

Theorem 3.1 ([25, Theorem 5.2.7]). Let $m \in L^{\infty}\left(\mathbb{R}^{n} \backslash\{0\}\right)$ be a complex-valued bounded function on $\mathbb{R}^{n} \backslash\{0\}$ that satisfies:

(a) Either the Mikhlin's condition

$$
\left|\partial_{\xi}^{\gamma} m(\xi)\right| \leq A|\xi|^{-|\gamma|}
$$

for all multi-indices $|\gamma| \leq\left[\frac{n}{2}\right]+1$.

(b) Or the Hörmander's condition

$$
\sup _{R>0} R^{-n+2|\gamma|} \int_{R<|\xi|<2 R}\left|\partial_{\xi}^{\gamma} m(\xi)\right|^{2} d \xi \leq A^{2}<\infty
$$




$$
\text { for all multi-indices }|\gamma| \leq\left[\frac{n}{2}\right]+1 \text {. }
$$

Then the operator $T_{m}$ whose symbol is $m$ is bounded in $L^{p}\left(\mathbb{R}^{n}\right)$ for any $p \in(1,+\infty)$, with an operator norm depending on $n, A, p,\|m\|_{\infty}$.

Remark 3.2. We point out there are sharper versions of the previous theorem replacing the Lebesgue spaces by Lorentz spaces (see e.g. [26]) and also more general variants (see e.g. [22]).

In our framework, we want to study boundedness properties of the multiplier $\Phi(-L)$ where

$$
\Phi(\lambda)=\int_{0}^{+\infty} G(+\infty, y) \partial_{y} \mathcal{K}(y, \lambda)^{2} a(y)^{2} d y .
$$

From now on and throughout the paper we will make the following standing assumptions on the functions involved in $\Phi$ :

- $a$ is bounded on $(0,+\infty)$;

- The functions $G(+\infty, y)$ and $G(z, y)$ defined in Lemma 2.2 are both of at most polynomial growth when $y, z \rightarrow+\infty$;

- The functions $\mathcal{K}$ and $\partial_{y} \mathcal{K}$ defined by (8) are both rapidly decreasing when $y, \lambda \rightarrow+\infty$.

To apply the previous theorem, we define $m(\xi)=\Phi\left(|\xi|^{2}\right)$ and to simplify the discussion we will consider only the Mikhlin's condition, since the conclusion of the discussion will be the same for Hörmander's case. It is clear that if $\mathcal{K}\left(y,|\xi|^{2}\right)$ satisfies a Mikhlin-type condition (i.e. enough derivatives in $\xi$ decay away from zero), then the Leibniz rule (together with dominated convergence) implies that so is $m$. Therefore, Theorem 3.1 gives that $\Phi(-L)$ is bounded on $L^{p}$ for every $p \in(1, \infty)$. Notice however that the constant in the estimate depends on the dimension $n$. In connection to other probabilistic (martingale) proofs of Theorem 3.1, we mention that in [34, Theorem 1.1], McConnell gave a probabilistic proof under the assumption that $|\gamma| \leq n+1$ in the context of functions taking values in a Banach spaces with the UMD (unconditional martingale difference sequence) property. There too, the constants depend on the dimension even in the case when the UMD space is $\mathbb{R}$.

In this section, we will prove a Gundy-Varopoulos representation for $\Phi(-L)$ therefore allowing us to prove the boundedness in $L^{p}$ with an explicit constant independent of the dimension (see Theorem 3.4). In some special cases of diffusion $\mathcal{B}$, one can compute explicitly $G$ and $\mathcal{K}$, allowing to check the previous decay assumptions and then leading to some special multipliers.

3.1. Construction of the martingale transform associated to a multiplier. We consider then the multiplier defined for $f \in C_{0}^{\infty}(M)$ by

$$
W f=\Phi(-L) f
$$

where

$$
\Phi(\lambda)=\int_{0}^{+\infty} G(+\infty, y) \partial_{y} \mathcal{K}(y, \lambda)^{2} a(y)^{2} d y .
$$

We again note that due to the growth assumptions on $G$ and $\partial_{y} \mathcal{K}$, the function $\Phi$ is bounded. Moreover, using the extension function $U_{f}$ defined in (7), one can see 
that $W$ satisfies the following integration by parts formula: for every $f, g \in C_{0}^{\infty}(M)$,

$$
\int_{M} g W f d \mu=\int_{0}^{+\infty} \int_{M} \partial_{y} U_{f}(y, x) \partial_{y} U_{g}(y, x) G(+\infty, y) a(y)^{2} d \mu(x) d y .
$$

Let now $\mathbb{P}^{x, y}$ be the probability measure associated with the stochastic process $\left(X_{t}, \eta_{t}\right)$ starting at the point $(x, y)$ with $x \in M$ and $y>0$, define a measure $\mathbb{P}^{y}$ by

$$
\mathbb{P}^{y}\left(\left(X_{t \wedge \tau}, \eta_{t \wedge \tau}\right) \in \Theta\right)=\int_{M} \mathbb{P}^{x, y}\left(\left(X_{t \wedge \tau}, \eta_{t \wedge \tau}\right) \in \Theta\right) d \mu(x)
$$

for any Borel set $\Theta \in M \times \mathbb{R}^{+}$. In particular, since $\mu$ is invariant for the semigroup for any Borel set $\Theta \in M, \mathbb{P}^{y}\left(X_{\tau} \in \Theta\right)=\mu(\Theta)$. From this it follows that for any nonnegative (or integrable function) $f$ on $M$, we have $\mathbb{E}^{y}\left(f\left(X_{\tau}\right)\right)=\int_{M} f(x) d \mu(x)$. In particular, for any $1<p<\infty$, the $L^{p}$ norm of the random variable $Z=$ $f\left(X_{\tau}\right)$ equals the $L^{p}(M, \mu)$ norm of the function $f$. In this case we will just write $\left\|f\left(X_{\tau}\right)\right\|_{L^{p}}=\|f\|_{L^{p}(M, \mu)}$.

Theorem 3.3. We have the following Gundy-Varopoulos type representation for $W$ : for every $f \in C_{0}^{\infty}(M)$ and $x \in M$,

$$
W f(x)=\frac{1}{2} \lim _{y_{0} \rightarrow+\infty} \mathbb{E}^{y_{0}}\left(e^{\int_{0}^{\tau} V\left(X_{u}\right) d u} \int_{0}^{\tau} e^{-\int_{0}^{s} V\left(X_{u}\right) d u} \partial_{y} U_{f}\left(X_{s}, \eta_{s}\right) a\left(\eta_{s}\right) d \beta_{s} \mid X_{\tau}=x\right) .
$$

Proof. Note first that as a consequence of Lemma 2.3, since $X$ and $\eta$ are independent, we have

$$
\mathbb{E}^{y}\left(\int_{0}^{\tau} F\left(X_{s}, \eta_{s}\right) d s\right)=\int_{0}^{+\infty} \int_{M} G(y, z) F(x, z) d \mu(x) d z,
$$

for all Borel function $F$ on $M \times \mathbb{R}^{+}$such that $\int_{M} \int_{0}^{\infty} \frac{h(z)}{a(z)^{2}}|F(x, z)| d z d \mu(x)<\infty$.

Let $f, g \in C_{0}^{\infty}(M)$. We observe that

$$
M_{\tau}^{g}=e^{\int_{0}^{\tau} V\left(X_{u}\right) d u} g\left(X_{\tau}\right) .
$$

Applying Itô's formula (9) for $M_{\tau}^{g}$ and the Itô isometry, one has

$$
\begin{aligned}
\int_{M} g(x) \mathbb{E}^{y_{0}} & \left(e^{\int_{0}^{\tau} V\left(X_{u}\right) d u} \int_{0}^{\tau} e^{-\int_{0}^{s} V\left(X_{u}\right) d u} \partial_{y} U_{f}\left(X_{s}, \eta_{s}\right) a\left(\eta_{s}\right) d \beta_{s} \mid X_{\tau}=x\right) d \mu(x) \\
& =\mathbb{E}^{y_{0}}\left(g\left(X_{\tau}\right) e^{\int_{0}^{\tau} V\left(X_{u}\right) d u} \int_{0}^{\tau} e^{-\int_{0}^{s} V\left(X_{u}\right) d u} \partial_{y} U_{f}\left(X_{s}, \eta_{s}\right) a\left(\eta_{s}\right) d \beta_{s}\right) \\
& =2 \mathbb{E}^{y_{0}}\left(\int_{0}^{\tau} \partial_{y} U_{g}\left(X_{s}, \eta_{s}\right) \partial_{y} U_{f}\left(X_{s}, \eta_{s}\right) a\left(\eta_{s}\right)^{2} d s\right) \\
& =2 \int_{0}^{+\infty} \int_{M} G\left(y_{0}, y\right) \partial_{y} U_{g}(x, y) \partial_{y} U_{f}(x, y) a(y)^{2} d \mu(x) d y
\end{aligned}
$$

where the last inequality is due to (11). Since $U_{f}(x, y)=\mathcal{K}(y,-L) f(x)$ and $L$ is self-adjoint, we have

$$
\int_{M} \partial_{y} U_{g}(x, y) \partial_{y} U_{f}(x, y) d \mu(x)=\int_{M} g(x) \partial_{y} \mathcal{K}(y,-L) \partial_{y} \mathcal{K}(y,-L) f(x) d \mu(x)
$$

and therefore

$$
\int_{0}^{+\infty} \int_{M} G\left(y_{0}, y\right) \partial_{y} U_{g}(x, y) \partial_{y} U_{f}(x, y) d \mu(x) a(y)^{2} d y
$$




$$
=\int_{M} g(x) \int_{0}^{+\infty} G\left(y_{0}, y\right) \partial_{y} \mathcal{K}(y,-L) \partial_{y} \mathcal{K}(y,-L) f(x) a(y)^{2} d y d \mu(x) .
$$

We conclude that for every $g \in C_{0}^{\infty}(M)$

$$
\begin{aligned}
& \int_{M} g(x) \mathbb{E}^{y_{0}}\left(e^{\int_{0}^{\tau} V\left(X_{u}\right) d u} \int_{0}^{\tau} e^{-\int_{0}^{s} V\left(X_{u}\right) d u} \partial_{y} U_{f}\left(X_{s}, \eta_{s}\right) a\left(\eta_{s}\right) d \beta_{s} \mid X_{\tau}=x\right) d \mu(x) \\
= & 2 \int_{M} g(x) \int_{0}^{+\infty} G\left(y_{0}, y\right) \partial_{y} \mathcal{K}(y,-L) \partial_{y} \mathcal{K}(y,-L) f(x) a(y)^{2} d y d \mu(x) .
\end{aligned}
$$

Therefore,

$$
\begin{aligned}
& \mathbb{E}^{y_{0}}\left(e^{\int_{0}^{\tau} V\left(X_{u}\right) d u} \int_{0}^{\tau} e^{-\int_{0}^{s} V\left(X_{u}\right) d u} \partial_{y} U_{f}\left(X_{s}, \eta_{s}\right) a\left(\eta_{s}\right) d \beta_{s} \mid X_{\tau}=x\right) \\
= & 2 \int_{0}^{+\infty} G\left(y_{0}, y\right) \partial_{y} \mathcal{K}(y,-L) \partial_{y} \mathcal{K}(y,-L) f(x) a(y)^{2} d y .
\end{aligned}
$$

The conclusion follows by taking the limit $y_{0} \rightarrow+\infty$.

\subsection{Boundedness in $L^{p}$.}

Theorem 3.4. The operator $W$ defined by (10) is bounded in $L^{p}$. Moreover, if the potential $V \equiv 0$, we have for every $f \in L^{p}(M, \mu)$

$$
\|W f\|_{p} \leq \frac{1}{2}\left(p^{*}-1\right)\|f\|_{p} .
$$

And, if the potential $V$ is not zero, then

$$
\|W f\|_{p} \leq \frac{3}{2}\left(p^{*}-1\right)\|f\|_{p} .
$$

Proof. Let $f \in C_{0}^{\infty}(M)$. One can write

$$
W f(x)=\frac{1}{2} \lim _{y_{0} \rightarrow+\infty} \mathbb{E}^{y_{0}}\left(e^{\int_{0}^{\tau} V\left(X_{u}\right) d u} \int_{0}^{\tau} e^{-\int_{0}^{s} V\left(X_{u}\right) d u} d Y_{s} \mid X_{\tau}=x\right),
$$

where

$$
Y_{t}=\int_{0}^{t} \partial_{y} U_{f}\left(X_{s}, \eta_{s}\right) a\left(\eta_{s}\right) d \beta_{s}
$$

If $V \equiv 0$, the martingale $Y$ is differentially subordinate to the martingale $U_{f}(X, \eta)$, see Lemma 2.5. One can conclude by using Theorem 2.7 with $V_{t}=0$.

Next we deal with the case $V \neq 0$ and adapt a method used in the proof of Theorem 1.1 in [8]. If $V \neq 0$, then $U_{f}(X, \eta)$ is not a martingale anymore. However, the martingale $Y$ is differentially subordinate to the martingale

$$
N_{t}:=\sum_{i=1}^{n} \int_{0}^{t \wedge \tau}\left(\sum_{j=1}^{n} v_{i j} \partial_{x_{j}}\right) U_{f}\left(X_{s}, \eta_{s}\right) d B_{s}^{i}+\int_{0}^{t \wedge \tau} \partial_{y} U_{f}\left(X_{s}, \eta_{s}\right) a\left(\eta_{s}\right) d \beta_{s},
$$

where we recall that $\left(v_{i j}(x)\right)$ is the square root of the matrix $\left(\sigma_{i j}(x)\right)$. From Itô's formula, one also has

$$
N_{t}=U_{f}\left(X_{t \wedge \tau}, \eta_{t \wedge \tau}\right)-U_{f}\left(X_{0}, \eta_{0}\right)-2 \int_{0}^{t \wedge \tau}(\Delta+\mathcal{B}) U_{f}\left(X_{s}, \eta_{s}\right) d s
$$

We now note that from Theorem 2.4

$$
(\Delta+\mathcal{B}) U_{f}=-V U_{f} .
$$


Therefore,

$$
N_{t}=U_{f}\left(X_{t \wedge \tau}, \eta_{t \wedge \tau}\right)-U_{f}\left(X_{0}, \eta_{0}\right)+2 \int_{0}^{t \wedge \tau} V\left(X_{s}\right) U_{f}\left(X_{s}, \eta_{s}\right) d s
$$

Suppose $f \geq 0$. Then, the above equality yields that $U_{f}\left(X_{t \wedge \tau}, \eta_{t \wedge \tau}\right)$ is a nonnegative sub-martingale. It follows from Lenglart-Lépingle-Pratelli [33, Theorem 3.2 , part 3)] that

$$
\left\|U_{f}\left(X_{0}, \eta_{0}\right)-2 \int_{0}^{\tau} V\left(X_{s}\right) U_{f}\left(X_{s}, \eta_{s}\right) d s\right\|_{p} \leq p\left\|U_{f}\left(X_{\tau}, \eta_{\tau}\right)\right\|_{p}=p\left\|f\left(X_{\tau}\right)\right\|_{p}=p\|f\|_{p},
$$

where we used the fact that the $L^{p}$ norm of the random variable $f\left(X_{\tau}\right)$ equals the $L^{p}(M, \mu)$ norm of the function $f$, as pointed earlier. For a general $f$, since $V$ is non-positive, we note that

$$
\left|U_{f}\left(X_{0}, \eta_{0}\right)-2 \int_{0}^{\tau} V\left(X_{s}\right) U_{f}\left(X_{s}, \eta_{s}\right) d s\right| \leq U_{|f|}\left(X_{0}, \eta_{0}\right)-2 \int_{0}^{\tau} V\left(X_{s}\right) U_{|f|}\left(X_{s}, \eta_{s}\right) d s .
$$

This yields that we always have

$$
\left\|N_{\tau}\right\|_{p} \leq(p+1)\|f\|_{p}
$$

Therefore by Lemma 2.7

$$
\left\|Y_{\tau}\right\|_{p} \leq\left(p^{*}-1\right)\left\|N_{\tau}\right\|_{p} \leq(p+1)\left(p^{*}-1\right)\|f\|_{p} .
$$

We conclude from the Gundy-Varopoulos type representation of $W$ and contraction of the conditional expectation that

$$
\|W f\|_{p} \leq \frac{1}{2}(p+1)\left(p^{*}-1\right)\|f\|_{p} .
$$

For $1<p \leq 2$, this gives the inequality in the statement of the theorem. The similar inequality in the range $p>2$ is obtained by using the fact that the $L^{p}$ adjoint operator of $W$ is itself.

3.3. Specific choices for the vertical diffusion. In this section we give explicit expressions of the operator $W$ depending upon the choices of the function $b$. It suffices to compute the function $\mathcal{K}(y, \lambda)$ defined in (8) and the Green function associated to the operator $\mathcal{B}$. For computations, it may be easier to use an alternative representation of the multiplier. Recall that

$$
\Phi(\lambda)=\int_{0}^{+\infty} G(+\infty, y) \partial_{y} \mathcal{K}(y, \lambda)^{2} a(y)^{2} d y .
$$

\section{Lemma 3.5.}

$$
\Phi(\lambda)=\frac{1}{2}-\lambda \int_{0}^{+\infty} G(+\infty, y) \mathcal{K}(y, \lambda)^{2} d y
$$

Proof. From the definition of $\mathcal{K}(y, \lambda)$ in (8), we have that $\mathcal{B K}(\cdot, \lambda)=\lambda \mathcal{K}(\cdot, \lambda)$, see page 18 in [19]. Moreover, $\mathcal{K}(y, \lambda)$ converges to 1 as $y \rightarrow 0$ and $\mathcal{K}(y, \lambda) \rightarrow 0$ as $y \rightarrow \infty$. Thus, using integration by parts one obtains

$$
\begin{aligned}
& \int_{0}^{+\infty} G(+\infty, y) \partial_{y} \mathcal{K}(y, \lambda)^{2} a(y)^{2} d y \\
& =\left.\mathcal{K}(y, \lambda) \partial_{y} \mathcal{K}(y, \lambda) G(+\infty, y) a(y)^{2}\right|_{0} ^{+\infty}-\int_{14}^{+\infty} \mathcal{K}(y, \lambda) \partial_{y}\left(a(y)^{2} G(+\infty, y) \partial_{y} \mathcal{K}(y, \lambda)\right) d y
\end{aligned}
$$




$$
\begin{aligned}
& =-\int_{0}^{+\infty} \mathcal{K}(y, \lambda) \partial_{y} \mathcal{K}(y, \lambda) d y-\int_{0}^{+\infty} \mathcal{K}(y, \lambda) G(+\infty, y) \mathcal{B} \mathcal{K}(y, \lambda) d y \\
& =\frac{1}{2}-\lambda \int_{0}^{+\infty} G(+\infty, y) \mathcal{K}(y, \lambda)^{2} d y .
\end{aligned}
$$

Here the second equality follows from the definition of $G(+\infty, y)$ which gives

$$
\partial_{y}\left(a(y)^{2} G(+\infty, y)\right)=\partial_{y}\left(h(y) \int_{0}^{y} \frac{d w}{h(w)}\right)=a(y)^{2} \frac{h^{\prime}(y)}{h(y)} G(+\infty, y)+1 .
$$

3.3.1. Brownian motion with negative drift. Assume that $a(y)=\sigma$ and $b(y)=$ $-2 m$, where $m \geq 0$ and $\sigma>0$. One computes that

$$
\mathcal{K}(y, \lambda)=e^{-\frac{y}{\sigma}\left(\sqrt{\lambda+\frac{m^{2}}{\sigma^{2}}}-\frac{m}{\sigma}\right)},
$$

see for instance [16, page 69] or [19, page 295]. Taking $h(x)=e^{-\frac{2 m}{\sigma^{2}}(x-1)}$ in Lemma 2.2 yields

$$
G(y, z)=\frac{1}{2 m} e^{-\frac{2 m}{\sigma^{2}} z}\left(e^{\frac{2 m}{\sigma^{2}}(y \wedge z)}-1\right) .
$$

In particular,

$$
G(+\infty, z)=\frac{1}{2 m}\left(1-e^{-\frac{2 m}{\sigma^{2}} z}\right)
$$

For this choice of $a$ and $b$, we can now rewrite the operator $W$ defined in (10) as

$$
W f=\frac{1}{4}\left(I-\frac{m}{\sigma}\left(-L+\frac{m^{2}}{\sigma^{2}}\right)^{-1 / 2}\right) f .
$$

We are now in position to conclude the proof of Theorem 1.1. We actually state a slightly stronger version including the potential $V$.

Theorem 3.6. Let $\Phi:[0,+\infty) \rightarrow \mathbb{C}$ be a bounded Borel function. If there exists a finite complex Borel measure $\alpha$ on $\mathbb{R}_{\geq 0}$ such that for every $x \in[0,+\infty)$,

$$
\Phi(x)=\int_{0}^{+\infty}\left(1-\frac{m}{\sqrt{m^{2}+x}}\right) d \alpha(m),
$$

then, for every $p>1$ and $f \in L^{p}(M, \mu)$,

$$
\|\Phi(-L) f\|_{p} \leq 6\left(p^{*}-1\right)|\alpha|\left(\mathbb{R}_{\geq 0}\right)\|f\|_{p} .
$$

If $V \equiv 0$, this bound can be improved to

$$
\|\Phi(-L) f\|_{p} \leq 2\left(p^{*}-1\right)|\alpha|\left(\mathbb{R}_{\geq 0}\right)\|f\|_{p} .
$$

Proof. It follows from the expression of $W$ and Theorem 3.4 that for every $m \geq 0$

$$
\left\|\left(I-m\left(-L+m^{2}\right)^{-1 / 2}\right) f\right\|_{p} \leq 6\left(p^{*}-1\right)\|f\|_{p} .
$$

Thus, we have

$$
\|\Phi(-L) f\|_{p} \leq 6\left(p^{*}-1\right)|\alpha|\left(\mathbb{R}_{\geq 0}\right)\|f\|_{p} .
$$

When $V \equiv 0$ the bound can be improved thanks to Theorem 3.4. 
3.3.2. Bessel processes. Assume that $a(y)=1$ and $b(y)=\frac{\gamma}{y}$, where $-1<\gamma<1$. Set $\gamma=1-2 s$, then one computes that

$$
\mathcal{K}_{s}(y, \lambda)=\frac{2^{1-s}}{\Gamma(s)} y^{s} \lambda^{s / 2} K_{s}\left(y \lambda^{1 / 2}\right)
$$

where $K_{s}(x)$ is the MacDonald function (Bessel function of the second kind) defined as follows

$$
K_{s}(x)=\frac{1}{2}\left(\frac{x}{2}\right)^{s} \int_{0}^{+\infty} \frac{e^{-t-\frac{x^{2}}{4 t}}}{t^{1+s}} d t .
$$

Taking $h(x)=y^{\gamma}$ in Lemma 2.2, it follows that $G(+\infty, y)=\frac{y}{2 s}$. Using the formula

$$
\int_{0}^{+\infty} y^{\alpha-1} K_{\nu}(y)^{2} d y=\frac{\sqrt{\pi}}{4 \Gamma\left(\frac{1+\alpha}{2}\right)} \Gamma\left(\frac{\alpha}{2}\right) \Gamma\left(\frac{\alpha}{2}-\nu\right) \Gamma\left(\frac{\alpha}{2}+\nu\right)
$$

that holds for $\alpha>2 \nu>0$ (see [1]), one gets $W f=\frac{1}{2(2-\gamma)} f$.

Remark 3.7 (Bessel processes with negative drift). Interestingly, some (partially) explicit computations may also be carried out in a class of processes extensively studied by Pitman 85 Yor in [37]. Those processes introduced by S. Watanabe in [43], and generalizing the Bessel processes, are sometimes called Bessel processes with negative (or descending) drift. Assume that $a(y)=1$ and

$$
b(y)=\frac{2 \nu+1}{y}-2 \delta \frac{K_{1+\nu}(\delta y)}{K_{\nu}(\delta y)}
$$

with $\nu, \delta>0$. In that case, direct computations show that the multiplier $W$ takes the form

$$
W=\frac{1}{2}+\frac{L\left(-L+\delta^{2}\right)^{\nu}}{\delta^{2 \nu}} \int_{0}^{+\infty} K_{\nu}\left(y \sqrt{-L+\delta^{2}}\right)^{2} \frac{I_{\nu}(\delta y)}{K_{\nu}(\delta y)} y d y
$$

\section{Generalized Riesz transforms}

In this section we will construct other operators arising from martingale transforms. We work with all the assumptions and notations of Section 2.1 but assume furthermore that the operator $\Delta$ admits a representation

$$
\Delta=-\sum_{i=1}^{n} \mathfrak{X}_{i}^{*} \mathfrak{X}_{i},
$$

where the $\mathfrak{X}_{i}$ 's are smooth vector fields on $M, \mathfrak{X}_{i}^{*}$ denotes the formal adjoint of $\mathfrak{X}_{i}$ with respect to $\mu$. We denote as before $L=\Delta+V$, where $V: M \rightarrow \mathbb{R}$ is as before the non-positive bounded smooth potential and $\left(P_{t}\right)_{t \geq 0}$ the heat semigroup with generator $L$. Note that we can write

$$
\Delta=\sum_{i=1}^{n} \mathfrak{X}_{i}^{2}+\mathfrak{X}_{0},
$$

for some smooth vector field $\mathfrak{X}_{0}$ and that from the celebrated Hörmander theorem [30], a sufficient condition for the local subellipticity of $\Delta$ is then that the vector fields $\mathfrak{X}_{0}, \mathfrak{X}_{1}, \cdots, \mathfrak{X}_{n}$ satisfy the bracket generating condition, see page 6 in [18]. 
Let $\left(X_{t}\right)_{t \geq 0}$ be the diffusion process on $M$ with generator $\sum_{i=1}^{n} \mathfrak{X}_{i}^{2}+\mathfrak{X}_{0}$ starting from the distribution $\mu$. We assume that $\left(X_{t}\right)_{t \geq 0}$ is nonexplosive and can be constructed via the Stratonovitch stochastic differential equation

$$
d X_{t}=\mathfrak{X}_{0}\left(X_{t}\right) d t+\sum_{i=1}^{n} \mathfrak{X}_{i}\left(X_{t}\right) \circ d B_{t}^{i}
$$

where $B_{t}=\left(B_{t}^{1}, \cdots, B_{t}^{n}\right)$ is a Brownian motion on $\mathbb{R}^{n}$ with generator $\Delta=\sum_{i=1}^{n} \frac{\partial^{2}}{\partial x_{i}^{2}}$.

Remark 4.1. For instance, if $M$ is a Euclidean space, then a standard sufficient condition so that $\left(X_{t}\right)_{t \geq 0}$ is nonexplosive and can be constructed via the above Stratonovitch stochastic differential equation is that the vector fields $\mathfrak{X}_{0}, \mathfrak{X}_{1}, \cdots, \mathfrak{X}_{n}$ have globally bounded derivatives, see for instance [17, Theorem 6.29]. In that case, if the vector fields $\mathfrak{X}_{0}, \mathfrak{X}_{1}, \cdots, \mathfrak{X}_{n}$ also satisfy the bracket generating condition, then $\Delta$ is locally subelliptic and essentially self-adjoint.

As before, see (6), we will consider the one-dimensional diffusion on $(0,+\infty)$ given by

$$
d \eta_{t}=b\left(\eta_{t}\right) d t+a\left(\eta_{t}\right) d \beta_{t}, \quad t<\tau
$$

where $\beta_{t}$ is a Brownian motion on $\mathbb{R}$ with $\mathbb{E}\left(\beta_{t}^{2}\right)=2 t$ which is independent from $\left(X_{t}\right)_{t \geq 0}$.

4.1. Operators arising from martingale transforms. We introduce now the class of operators under consideration. For any $1 \leq i, j \leq n$, we consider the operators

$$
T_{i} f=\int_{0}^{+\infty} a(y) G(+\infty, y) \partial_{y} \mathcal{K}(y,-L) \mathfrak{X}_{i} \mathcal{K}(y,-L) f d y
$$

and

$$
S_{i j} f=\int_{0}^{+\infty} G(+\infty, y) \mathcal{K}(y,-L) \mathfrak{X}_{j}^{*} \mathfrak{X}_{i} \mathcal{K}(y,-L) f d y .
$$

Theorem 4.2. We have the following Gundy-Varopoulos type representations: for every $f \in C_{0}^{\infty}(M)$ and $x \in M$

$$
\begin{aligned}
T_{i} f(x) & =\frac{1}{2} \lim _{y_{0} \rightarrow+\infty} \mathbb{E}^{y_{0}}\left(e^{\int_{0}^{\tau} V\left(X_{u}\right) d u} \int_{0}^{\tau} e^{-\int_{0}^{s} V\left(X_{u}\right) d u} \mathfrak{X}_{i} U_{f}\left(X_{s}, \eta_{s}\right) d \beta_{s} \mid X_{\tau}=x\right), \\
S_{i j} f(x) & =\frac{1}{2} \lim _{y_{0} \rightarrow+\infty} \mathbb{E}^{y_{0}}\left(e^{\int_{0}^{\tau} V\left(X_{u}\right) d u} \int_{0}^{\tau} e^{-\int_{0}^{s} V\left(X_{u}\right) d u} \mathfrak{X}_{i} U_{f}\left(X_{s}, \eta_{s}\right) d B_{s}^{j} \mid X_{\tau}=x\right) .
\end{aligned}
$$

Proof. The proof is similar to the proof of Theorem 3.3, we present it for completeness. It suffices to show the first expression of $T_{i}$. The same proof also works for $S_{i j}$. Let $f, g \in C_{0}^{\infty}(M)$. We recall $M_{\tau}^{g}=e^{\int_{0}^{\tau} V\left(X_{u}\right) d u} g\left(X_{\tau}\right)$. Applying Itô's formula for $M_{t}^{g}$ gives that

$$
\begin{aligned}
M_{t}^{g}=U_{g}\left(X_{0}, \eta_{0}\right) & +\sum_{i=1}^{n} \int_{0}^{t \wedge \tau} e^{\int_{0}^{s} V\left(X_{u}\right) d u} \mathfrak{X}_{i} U_{g}\left(X_{s}, \eta_{s}\right) d B_{s}^{i} \\
& +\int_{0}^{t \wedge \tau} e^{\int_{0}^{s} V\left(X_{u}\right) d u} \partial_{y} U_{g}\left(X_{s}, \eta_{s}\right) a\left(\eta_{s}\right) d \beta_{s} .
\end{aligned}
$$

By the Itô isometry, one has

$$
\int_{M} g(x) \mathbb{E}^{y_{0}}\left(e^{\int_{0}^{\tau} V\left(X_{u}\right) d u} \int_{0}^{\tau} e^{-\int_{0}^{s} V\left(X_{u}\right) d u} \mathfrak{X}_{i} U_{f}\left(X_{s}, \eta_{s}\right) d \beta_{s} \mid X_{\tau}=x\right) d \mu(x)
$$




$$
\begin{aligned}
& =\mathbb{E}^{y_{0}}\left(g\left(X_{\tau}\right) e^{\int_{0}^{\tau} V\left(X_{u}\right) d u} \int_{0}^{\tau} e^{-\int_{0}^{s} V\left(X_{u}\right) d u} \mathfrak{X}_{i} U_{f}\left(X_{s}, \eta_{s}\right) d \beta_{s}\right) \\
& =2 \mathbb{E}^{y_{0}}\left(\int_{0}^{\tau} \partial_{y} U_{g}\left(X_{s}, \eta_{s}\right) \mathfrak{X}_{i} U_{f}\left(X_{s}, \eta_{s}\right) a\left(\eta_{s}\right) d s\right) \\
& =2 \int_{0}^{+\infty} \int_{M} a(y) G\left(y_{0}, y\right) \partial_{y} U_{g}(x, y) \mathfrak{X}_{i} U_{f}(x, y) d \mu(x) d y,
\end{aligned}
$$

where the last equality is due to $(11)$. Since $U_{f}(x, y)=\mathcal{K}(y,-L) f(x)$ and $L$ is self-adjoint, then

$$
\int_{M} \partial_{y} U_{g}(x, y) \mathfrak{X}_{i} U_{f}(x, y) d \mu(x)=\int_{M} g(x) \partial_{y} \mathcal{K}(y,-L) \mathfrak{X}_{i} \mathcal{K}(y,-L) f(x) d \mu(x)
$$

and

$$
\begin{aligned}
\int_{0}^{+\infty} \int_{M} a(y) G\left(y_{0}, y\right) \partial_{y} U_{g}(x, y) \mathfrak{X}_{i} U_{f}(x, y) d \mu(x) d y \\
=\int_{M} g(x) \int_{0}^{+\infty} a(y) G\left(y_{0}, y\right) \partial_{y} \mathcal{K}(y,-L) \mathfrak{X}_{i} \mathcal{K}(y,-L) f(x) d y d \mu(x) .
\end{aligned}
$$

The rest of the proof thus immediately follows.

\subsection{Boundedness in $L^{p}(\mu)$.}

Corollary 4.3. Let $1 \leq i \leq n$. For all $1<p<\infty$ and all $f \in C_{0}^{\infty}(M)$ we have

$$
\left\|T_{i} f\right\|_{p} \leq \frac{3}{2}\left(p^{*}-1\right)\|f\|_{p} .
$$

Moreover, if the potential $V \equiv 0$, then

$$
\left\|T_{i} f\right\|_{p} \leq \frac{1}{2}\left(p^{*}-1\right)\|f\|_{p} .
$$

Proof. When $V \equiv 0$, the operator $T_{i}$ can be rewritten as

$$
T_{i} f(x)=\frac{1}{2} \lim _{y_{0} \rightarrow+\infty} \mathbb{E}^{y_{0}}\left(\int_{0}^{\tau} A_{i}\left(\mathfrak{X}, \partial_{y}\right)^{T} U_{f}\left(X_{s}, \eta_{s}\right) \cdot\left(d B_{s}, d \beta_{s}\right) \mid X_{\tau}=x\right),
$$

where $A_{i}$ is an $(n+1) \times(n+1)$ matrix with the entry $a_{(n+1), i}=1$ and otherwise 0 . Notice that the martingale $\int_{0}^{t \wedge \tau} A_{i}\left(\mathfrak{X}, \partial_{y}\right)^{T} U_{f}\left(X_{s}, \eta_{s}\right) \cdot\left(d B_{s}, d \beta_{s}\right)$ is differentially subordinate to the martingale $U_{f}\left(X_{t \wedge \tau}, \eta_{t \wedge \tau}\right)$. It follows from Theorem 2.6 that

$$
\left\|T_{i} f\right\|_{p} \leq \frac{1}{2}\left(p^{*}-1\right)\|f\|_{p}
$$

When $V \neq 0$, then the same method as for the proof of Theorem 3.4 implies the desired estimate.

Corollary 4.4. Let $1 \leq i, j \leq n$. For all $1<p<\infty$ and all $f \in C_{0}^{\infty}(M)$ we have

$$
\left\|S_{i j} f\right\|_{p} \leq \frac{3}{2}\left(p^{*}-1\right)\|f\|_{p} .
$$

Moreover, if the potential $V \equiv 0$, then

$$
\left\|S_{i j} f\right\|_{p} \leq \frac{1}{2}\left(p^{*}-1\right)\|f\|_{p} .
$$


Proof. Similarly as for $T_{i}$, when $V \equiv 0$ one can write $S_{i j}$ as

$$
S_{i j} f(x)=\frac{1}{2} \lim _{y_{0} \rightarrow+\infty} \mathbb{E}^{y_{0}}\left(\int_{0}^{\tau} A_{i j}\left(\mathfrak{X}, \partial_{y}\right)^{T} U_{f}\left(X_{s}, \eta_{s}\right) \cdot\left(d B_{s}, d \beta_{s}\right) \mid X_{\tau}=x\right),
$$

where $A_{i j}$ is an $(n+1) \times(n+1)$ matrix with the entries $a_{i, j}=1$ and otherwise 0 . Since $\int_{0}^{t \wedge \tau} A_{i}\left(\mathfrak{X}, \partial_{y}\right)^{T} U_{f}\left(X_{s}, \eta_{s}\right) \cdot\left(d B_{s}, d \beta_{s}\right)$ is differentially subordinate to $U_{f}\left(X_{t \wedge \tau}, \eta_{t \wedge \tau}\right)$, then Theorem 2.6 yields

$$
\left\|S_{i j} f\right\|_{p} \leq \frac{1}{2}\left(p^{*}-1\right)\|f\|_{p}
$$

4.3. Euclidean spaces and Lie groups of compact type. We now apply our results to the case of Euclidean spaces and Lie groups of compact type. In those cases, for the transforms we are interested in, the operators $\mathfrak{X}_{i}$ 's and $\mathfrak{X}_{i}^{*}$ 's do commute with $L$. As a consequence, one has

$$
T_{i} f=\int_{0}^{+\infty} a(y) G(+\infty, y) \partial_{y} \mathcal{K}(y,-L) \mathcal{K}(y,-L) \mathfrak{X}_{i} f d y
$$

and

$$
S_{i j} f=\int_{0}^{+\infty} G(+\infty, y) \mathcal{K}(y,-L)^{2} \mathfrak{X}_{j}^{*} \mathfrak{X}_{i} f d y .
$$

4.3.1. Brownian motion with negative drift as vertical diffusion. Consider the Euclidean spaces $\mathbb{R}^{n}$. We assume that the potential $V$ in the operator $L$ is null. In this case, $\mathfrak{X}_{i}=\partial_{x_{i}}$ commutes with the Laplace operator $L=\Delta$.

Lemma 4.5. Let $1 \leq i, j \leq n$ and $\sigma>0, m \geq 0$. For the choice $\mathcal{B}=\sigma^{2} \frac{\partial^{2}}{\partial y^{2}}-2 m \frac{\partial}{\partial y}$, one has

$$
T_{i} f=-\frac{1}{4}\left(-\Delta+\frac{m^{2}}{\sigma^{2}}\right)^{-1 / 2} \partial_{x_{i}} f
$$

and

$$
S_{i j} f=-\frac{1}{4}\left(\sqrt{-\Delta+\frac{m^{2}}{\sigma^{2}}}-\frac{m}{\sigma}\right)^{-1}\left(-\Delta+\frac{m^{2}}{\sigma^{2}}\right)^{-1 / 2} \partial_{x_{i}} \partial_{x_{j}} f .
$$

Proof. Since $\partial_{x_{i}}$ commutes with $\Delta$, the operator $T_{i}$ becomes

$$
\begin{aligned}
T_{i} f & =\left(\int_{0}^{+\infty} \frac{1-e^{\frac{2 m}{\sigma^{2}} y}}{2 m}\left(\sqrt{-\Delta+\frac{m^{2}}{\sigma^{2}}}-\frac{m}{\sigma}\right) e^{-\frac{2 y}{\sigma} \sqrt{-\Delta+\frac{m^{2}}{\sigma^{2}}}} d y\right) \partial_{x_{i}} f \\
& =\frac{1}{2 m}\left(\sqrt{-\Delta+\frac{m^{2}}{\sigma^{2}}}-\frac{m}{\sigma}\right)\left(\frac{\sigma}{2}\left(\sqrt{-\Delta+\frac{m^{2}}{\sigma^{2}}}\right)^{-1}-\left(\frac{2}{\sigma} \sqrt{-\Delta+\frac{m^{2}}{\sigma^{2}}}-\frac{2 m}{\sigma^{2}}\right)^{-1}\right) \\
& =\frac{\sigma}{4 m}\left(\left(\sqrt{-\Delta+\frac{m^{2}}{\sigma^{2}}}-\frac{m}{\sigma}\right)\left(\sqrt{-\Delta+\frac{m^{2}}{\sigma^{2}}}\right)^{-1}-I\right) \partial_{x_{i}} f \\
& =-\frac{1}{4}\left(-\Delta+\frac{m^{2}}{\sigma^{2}}\right)^{-1 / 2} \partial_{x_{i}} f .
\end{aligned}
$$


Similarly, we have

$$
\begin{aligned}
S_{i j} f & =\left(\int_{0}^{+\infty} \frac{1-e^{\frac{2 m}{\sigma^{2}} y}}{2 m} e^{-\frac{2 y}{\sigma} \sqrt{-\Delta+\frac{m^{2}}{\sigma^{2}}}} d y\right) \partial_{x_{i}} \partial_{x_{j}} f \\
& =-\frac{\sigma}{4 m}\left(\left(\sqrt{-\Delta+\frac{m^{2}}{\sigma^{2}}}-\frac{m}{\sigma}\right)^{-1}-\left(\sqrt{-\Delta+\frac{m^{2}}{\sigma^{2}}}\right)^{-1}\right) \partial_{x_{i}} \partial_{x_{j}} f \\
& =-\frac{1}{4}\left(\sqrt{-\Delta+\frac{m^{2}}{\sigma^{2}}}-\frac{m}{\sigma}\right)^{-1}\left(-\Delta+\frac{m^{2}}{\sigma^{2}}\right)^{-1 / 2} \partial_{x_{i}} \partial_{x_{j}} f .
\end{aligned}
$$

We obtain therefore:

Proposition 4.6. Let $1 \leq i, j \leq n$ and $m \geq 0, \sigma>0$. Then

$$
\begin{gathered}
\left\|\left(-\Delta+\frac{m^{2}}{\sigma^{2}}\right)^{-1 / 2} \partial_{x_{i}} f\right\|_{p} \leq \cot \left(\frac{\pi}{2 p^{*}}\right)\|f\|_{p}, \\
\left\|\left(\sqrt{-\Delta+\frac{m^{2}}{\sigma^{2}}}-\frac{m}{\sigma}\right)^{-1}\left(-\Delta+\frac{m^{2}}{\sigma^{2}}\right)^{-1 / 2} \partial_{x_{i}} \partial_{x_{j}} f\right\|_{p} \leq\left(p^{*}-1\right)\|f\|_{p} .
\end{gathered}
$$

Proof. Recall the Gundy-Varopoulos type representation of $T_{i}$ in Theorem 4.2:

$$
T_{i} f(x)=\frac{1}{2} \lim _{y_{0} \rightarrow+\infty} \mathbb{E}^{y_{0}}\left(\int_{0}^{\tau} \partial_{x_{i}} U_{f}\left(X_{s}, \eta_{s}\right) d \beta_{s} \mid X_{\tau}=x\right) .
$$

In a similar way we also have

$$
T_{i} f(x)=-\frac{1}{2} \lim _{y_{0} \rightarrow+\infty} \mathbb{E}^{y_{0}}\left(\int_{0}^{\tau} \sigma \partial_{y} U_{f}\left(X_{s}, \eta_{s}\right) d B_{s}^{i} \mid X_{\tau}=x\right) .
$$

To see this, observe that by Itô's formula (12) and the Itô isometry, one has for any $y_{0}>0$

$$
\begin{aligned}
\int_{M} g(x) \mathbb{E}^{y_{0}} & \left(\int_{0}^{\tau} \sigma \partial_{y} U_{f}\left(X_{s}, \eta_{s}\right) d B_{s}^{i} \mid X_{\tau}=x\right) d \mu(x)=\mathbb{E}^{y_{0}}\left(g\left(X_{\tau}\right) \int_{0}^{\tau} \sigma \partial_{y} U_{f}\left(X_{s}, \eta_{s}\right) d B_{s}^{i}\right) \\
& =2 \mathbb{E}^{y_{0}}\left(\int_{0}^{\tau} \sigma \partial_{x_{i}} U_{g}\left(X_{s}, \eta_{s}\right) \partial_{y} U_{f}\left(X_{s}, \eta_{s}\right) d s\right) \\
& =2 \int_{0}^{+\infty} \int_{M} \sigma G\left(y_{0}, y\right) \partial_{x_{i}} U_{g}(x, y) \partial_{y} U_{f}(x, y) d \mu(x) d y \\
& =-2 \int_{M} g(x) \int_{0}^{+\infty} \sigma G\left(y_{0}, y\right) \partial_{y} \mathcal{K}(y,-L) \partial_{x_{i}} \mathcal{K}(y,-L) f(x) d y d \mu(x) .
\end{aligned}
$$

Taking the limit $y_{0} \rightarrow \infty$ gives the second expression of $T_{i} f$. Therefore

$$
T_{i} f(x)=\frac{1}{4} \lim _{y_{0} \rightarrow+\infty} \mathbb{E}^{y_{0}}\left(\int_{0}^{\tau} A_{i}\left(\mathfrak{X}, \partial_{y}\right)^{T} U_{f}\left(X_{s}, \eta_{s}\right) \cdot\left(d B_{s}, d \beta_{s}\right) \mid X_{\tau}=x\right),
$$

where $A_{i}$ is an $(n+1) \times(n+1)$ matrix with the entries $a_{(n+1), i}=1, a_{i,(n+1)}=-\sigma$ and otherwise 0 .

With this matrix we claim that (1) the martingale $N_{t}:=\int_{0}^{t \wedge \tau} A_{i}\left(\mathfrak{X}, \partial_{y}\right)^{T} U_{f}\left(X_{s}, \eta_{s}\right)$. $\left(d B_{s}, d \beta_{s}\right)$ is differentially subordinate to $M_{t}^{f}=U_{f}\left(X_{t \wedge \tau}, \eta_{t \wedge \tau}\right)$ and that (2) the 
two martingales are orthogonal. That is, $\left\langle N, M^{f}\right\rangle_{t}=0$. To verify (1) recall that that

$$
M_{t}^{f}=f\left(X_{0}, \eta_{0}\right)+\sum_{j=1}^{n} \int_{0}^{t \wedge \tau} \partial_{x_{j}} U_{f}\left(X_{s}, \eta_{s}\right) d B_{s}^{j}+\int_{0}^{t \wedge \tau} \partial_{y} U_{f}\left(X_{s}, \eta_{s}\right) \sigma d \beta_{s},
$$

and

$$
\left\langle M^{f}\right\rangle_{t}=2 \sum_{j=1}^{n} \int_{0}^{t \wedge \tau}\left(\partial_{x_{j}} U_{f}\left(X_{s}, \eta_{s}\right)\right)^{2} d s+2 \int_{0}^{t \wedge \tau}\left(\partial_{y} U_{f}\left(X_{s}, \eta_{s}\right)\right)^{2} \sigma^{2} d s
$$

Similarly,

$$
N_{t}=\int_{0}^{t \wedge \tau} \partial_{x_{i}} U_{f}\left(X_{s}, \eta_{s}\right) d \beta_{s}-\int_{0}^{t \wedge \tau} \partial_{y} U_{f}\left(X_{s}, \eta_{s}\right) \sigma d B_{s}^{i}
$$

and

$$
\langle N\rangle_{t}=2 \int_{0}^{t \wedge \tau}\left(\partial_{x_{i}} U_{f}\left(X_{s}, \eta_{s}\right)\right)^{2} d s+2 \int_{0}^{t \wedge \tau}\left(\partial_{y} U_{f}\left(X_{s}, \eta_{s}\right)\right)^{2} \sigma^{2} d s
$$

Thus

$$
\left\langle M^{f}\right\rangle_{t}-\langle N\rangle_{t}=2 \sum_{j \neq i} \int_{0}^{t \wedge \tau}\left(\partial_{x_{j}} U_{f}\left(X_{s}, \eta_{s}\right)\right)^{2} d s,
$$

which is a nondecreasing and nonnegative function of $t$. This proves the differential subordination property.

To prove the orthogonality we note that

$$
\begin{aligned}
\left\langle N, M^{f}\right\rangle_{t}= & -\sigma \int_{0}^{t \wedge \tau} \partial_{x_{i}} U_{f}\left(X_{s}, \eta_{s}\right) \partial_{y} U_{f}\left(X_{s}, \eta_{s}\right) d\left\langle B^{i}\right\rangle_{s} \\
& +\sigma \int_{0}^{t \wedge \tau} \partial_{y} U_{f}\left(X_{s}, \eta_{s}\right) \partial_{x_{i}} U_{f}\left(X_{s}, \eta_{s}\right) d\langle\beta\rangle_{s} \\
= & 0 .
\end{aligned}
$$

We can now apply (15) and the martingale inequality in Theorem 2.6. It follows that

$$
\left\|T_{i} f\right\|_{p} \leq \frac{1}{4} \cot \left(\frac{\pi}{2 p^{*}}\right)\|f\|_{p}
$$

and hence by Lemma 4.5,

$$
\left\|\left(-\Delta+\frac{m^{2}}{\sigma^{2}}\right)^{-1 / 2} \partial_{x_{i}} f\right\|_{p}=4\left\|T_{i} f\right\|_{p} \leq \cot \left(\frac{\pi}{2 p^{*}}\right)\|f\|_{p} .
$$

On the other hand

$$
\begin{aligned}
S_{i j} f(x) & =\frac{1}{2} \lim _{y_{0} \rightarrow+\infty} \mathbb{E}^{y_{0}}\left(\int_{0}^{\tau} \partial_{x_{j}} U_{f}\left(X_{s}, \eta_{s}\right) d B_{s}^{i} \mid X_{\tau}=x\right) \\
& =\frac{1}{4} \lim _{y_{0} \rightarrow+\infty} \mathbb{E}^{y_{0}}\left(\int_{0}^{\tau} A_{i j}\left(\mathfrak{X}, \partial_{y}\right)^{T} U_{f}\left(X_{s}, \eta_{s}\right) \cdot\left(d B_{s}, d \beta_{s}\right) \mid X_{\tau}=x\right),
\end{aligned}
$$

where $A_{i j}$ is an $(n+1) \times(n+1)$ matrix with the entries $a_{i, j}=a_{j, i}=1$ and otherwise 0 . Observe that the matrix norm of $A_{i j}$ is 1 , then the martingale $\int_{0}^{t \wedge \tau} A_{i j}\left(\mathfrak{X}, \partial_{y}\right)^{T} U_{f}\left(X_{s}, \eta_{s}\right) \cdot\left(d B_{s}, d \beta_{s}\right)$ is differentially subordinate to $U_{f}\left(X_{t \wedge \tau}, \eta_{t \wedge \tau}\right)$. 
It follows from Theorem 2.6 that

$$
\left\|S_{i j} f\right\|_{p} \leq \frac{1}{4}\left(p^{*}-1\right)\|f\|_{p}
$$

and again by Lemma 4.5 ,

$$
\left\|\left(\sqrt{-\Delta+\frac{m^{2}}{\sigma^{2}}}-\frac{m}{\sigma}\right)^{-1}\left(-\Delta+\frac{m^{2}}{\sigma^{2}}\right)^{-1 / 2} \partial_{x_{i}} \partial_{x_{j}} f\right\|_{p} \leq\left(p^{*}-1\right)\|f\|_{p} .
$$

Remark 4.7. The degenerate case $\sigma=0$ corresponds to the case where $d \eta_{t}=$ $-2 m d t$, i.e. $\eta_{t}=2 m(T-t)$, where $T=\tau$ is deterministic. This gives the spacetime process introduced in [5]. That is, taking the limit $\sigma \rightarrow 0$ in (14) with $m>0$ fixed, we recover the main results in [5] and [42] concerning the norms of second order Riesz transforms. Namely that

$$
\left\|2 R_{i} R_{j}\right\|_{p}=\left\|2(-\Delta)^{-1} \partial_{x_{i}} \partial_{x_{j}}\right\|_{p} \leq\left(p^{*}-1\right)\|f\|_{p} .
$$

Indeed, formally as $\sigma \rightarrow 0$ one has

$$
\left(\sqrt{-\Delta+\frac{m^{2}}{\sigma^{2}}}-\frac{m}{\sigma}\right)^{-1}\left(-\Delta+\frac{m^{2}}{\sigma^{2}}\right)^{-1 / 2} \partial_{x_{i}} \partial_{x_{j}} f \rightarrow 2(-\Delta)^{-1} \partial_{x_{i}} \partial_{x_{j}} f .
$$

In the same way, we obtain that

$$
\left\|R_{i}^{2}-R_{j}^{2}\right\|_{p}=\left\|(-\Delta)^{-1} \partial_{x_{i}} \partial_{x_{i}}-(-\Delta)^{-1} \partial_{x_{j}} \partial_{x_{j}}\right\|_{p} \leq\left(p^{*}-1\right)\|f\|_{p} .
$$

For $i \neq j$, the bounds $\left(p^{*}-1\right)$ in (16) and (17) were shown to be the best possible in [24].

When $i=j$, combining our methods here with the martingale inequalities from [11] we would obtain (again by letting $\sigma \rightarrow 0$ ) the inequality

$$
\left\|R_{i}^{2} f\right\|_{p}=\left\|(-\Delta)^{-1} \partial_{x_{i}} \partial_{x_{i}}\right\|_{p} \leq c_{p}\|f\|_{p}
$$

first proved in [11]. Here, $c_{p}$ is the best constant found in [21] for non-symmetric martingale transforms, i.e. martingale transforms where the predictable sequences take values in $[0,1]$. Although the constant is not as nice as Burkholder's $\left(p^{*}-1\right)$ for general martingale transforms, it can be estimated quite well and in particular it satisfies for large $p$,

$$
c_{p} \approx \frac{p}{2}+\frac{1}{2} \log \left(\frac{1+e^{-2}}{2}\right)+\frac{\alpha_{2}}{p}
$$

where

$$
\alpha_{2}=\left[\log \left(\frac{1+e^{-2}}{2}\right)\right]^{2}+\frac{1}{2} \log \left(\frac{1+e^{-2}}{2}\right)-2\left(\frac{e^{-2}}{1+e^{-2}}\right)^{2} .
$$

In addition it follows easily from Burkholder's inequality (see [21, Theorem 4.1]) that the constant $c_{p}$ satisfies the bounds

$$
\max \left(1, \frac{p^{*}}{2}-1\right) \leq c_{p} \leq \frac{p^{*}}{2} .
$$

It is also important to mention here that the constant $c_{p}$ in (18) is also best possible. This was first proved in [11]. For this and more general results related to second order Riesz transforms, we refer the reader to [11] and particularly Theorems 1.4, 1.5, and Corollary 1.3. 
On the complex plane $\mathbb{C}$, which we identify with $\mathbb{R}^{2}$, the Beurling-Ahlfors operator is defined by $B f=(-\Delta)^{-1} \partial^{2} f$, where $\partial$ is the Cauchy-Riemann operator $\partial f=\frac{\partial f}{\partial x_{1}}-i \frac{\partial f}{\partial x_{2}}$. A longstanding open problem with connections to several areas of analysis, PDE's and geometry, known as Iwaniec's conjecture [3, p.129], asserts that

$$
\|B f\|_{p} \leq\left(p^{*}-1\right)\|f\|_{p}, \quad 1<p<\infty
$$

for all $f: \mathbb{C} \rightarrow \mathbb{C}, f \in C_{0}^{\infty}(\mathbb{C})$.

That the constant $\left(p^{*}-1\right)$ in (19) cannot be improved has been known for many years, see [3]. Writing the operator $B=R_{1}^{2}-R_{2}^{2}+2 i R_{1} R_{2}$ in terms of Riesz transforms we see from the above discussion that the real and imaginary parts of the Beurling-Ahlfors operator have the same norm as the martingale transforms, that is, the Burkholder constant $p^{*}-1$. This is in fact the same for functions taking values in a Banach space with the UMD property, see [24, Theorem 1.1]. Applying (16) and (17) leads to the estimate $\|B f\|_{p} \leq 2\left(p^{*}-1\right)\|f\|_{p}$. This bound was first proved in [42] and [5] and later improved to $1.575\left(p^{*}-1\right)$ in [10] by martingale inequalties applied to martingales satisfying certain orthogonality properties. For a detailed discussion of these results we refer the reader to [6]. The key point in [5] and [10] is to use the martingale techniques applied to the space-time process. That is, build the martingales on the process $\left(X_{t}, T-t\right)$ which arise from the heat extension rather than the Poisson extension. Given that we now know that the process $\left(X_{t}, T-t\right)$ arises from the general Poison extensions treated in this paper by letting $\sigma \rightarrow 0$, it is natural to wonder if further progress on Iwaniec's conjecture can be made by better choices of the vertical diffusion $\eta_{t}$.

It is also interesting to note that as $\sigma \rightarrow+\infty$ (or equivalently $m \rightarrow 0$ ), we get the inequality

$$
\left\|(-\Delta)^{-1 / 2} \partial_{x_{i}} f\right\|_{p} \leq \cot \left(\frac{\pi}{2 p^{*}}\right)\|f\|_{p},
$$

which is sharp as shown in [31] and [13]. Thus, the inequalities (13) and (14) are both sharp in the sense that there is no universal constant $C<1$ independent of $\sigma$ and $m$ for which the first holds with $C \cot \left(\frac{\pi}{2 p^{*}}\right)$ on the right hand side and the second with $C\left(p^{*}-1\right)$.

The previous methods can be applied to Lie groups of compact type. Let $G$ be a Lie group of compact type with Lie algebra $\mathfrak{g}$. We recall that $G$ is called a Lie group of compact type if its Lie algebra $\mathfrak{g}$ admits an Ad-invariant inner product. In that case, this equips $G$ with a bi-invariant metric. Note that Euclidean spaces are examples of Lie groups of compact type so that this framework is a generalization of the Euclidean one.

We consider an orthonormal basis $\mathfrak{X}_{1}, \cdots, \mathfrak{X}_{n}$ of $\mathfrak{g}$. In this setting the LaplaceBeltrami operator can be written as

$$
L=\sum_{i=1}^{n} \mathfrak{X}_{i}^{2}
$$

Observe that $L$ is essentially self-adjoint on the space of smooth and compactly supported functions. Moreover, $\mathfrak{X}_{i}^{*}=-\mathfrak{X}_{i}$ and $\mathfrak{X}_{i}$ commutes with $L$. In the same manner as Euclidean spaces case we obtain the following result. 
Proposition 4.8. Let $G$ be a Lie group of compact type endowed with a bi-invariant Riemannian structure. Let $1 \leq i, j \leq n$ and $m \geq 0$. Then

$$
\begin{gathered}
\left\|\left(-L+m^{2}\right)^{-1 / 2} \mathfrak{X}_{i} f\right\|_{p} \leq \cot \left(\frac{\pi}{2 p^{*}}\right)\|f\|_{p}, \\
\left\|\left(\sqrt{-L+m^{2}}-m\right)^{-1}\left(-L+m^{2}\right)^{-1 / 2} \frac{1}{2}\left(\mathfrak{X}_{i} \mathfrak{X}_{j}+\mathfrak{X}_{j} \mathfrak{X}_{i}\right) f\right\|_{p} \leq\left(p^{*}-1\right)\|f\|_{p} .
\end{gathered}
$$

The proof of Theorem 1.2 easily follows from Proposition 4.8 by integrating with respect to $m$.

4.3.2. Bessel process as vertical diffusion. In this section, we work on Lie groups of compact type endowed with bi-invariant Riemannian structures.

Lemma 4.9. Let $G$ be a Lie group of compact type endowed with a bi-invariant Riemannian structure. Let $1 \leq i, j \leq n$. For the choice $\mathcal{B}=\frac{\partial^{2}}{\partial y^{2}}+b(y) \frac{\partial}{\partial y}$ with $b(y)=\frac{\gamma}{y},-1<\gamma<1$, one has

$$
T_{i} f=-\frac{\pi^{2} \Gamma(4 s)}{2^{8 s} s^{2} \Gamma(s)^{4}}(-L)^{-1 / 2} \mathfrak{X}_{i}
$$

and

where $\gamma=1-2 s$.

$$
S_{i j} f=\frac{s}{2 s+1}(-L)^{-1} \mathfrak{X}_{i} \mathfrak{X}_{j},
$$

Proof. Using the Bessel process, we recall that

$$
\mathcal{K}_{s}(y, \lambda)=\frac{2^{1-s}}{\Gamma(s)} y^{s} \lambda^{s / 2} K_{s}\left(y \lambda^{1 / 2}\right)
$$

with $\gamma=1-2 s$ and that $G(+\infty, y)=\frac{y}{2 s}$. Therefore

$$
\begin{aligned}
\int_{0}^{+\infty} G(+\infty, y) \partial_{y} \mathcal{K}(y, \lambda) \mathcal{K}(y, \lambda) d y & =\frac{1}{2} \int_{0}^{+\infty} G(+\infty, y) \partial_{y}\left(\mathcal{K}(y, \lambda)^{2}\right) d y \\
& =-\frac{1}{4 s} \frac{2^{2-2 s}}{\Gamma(s)^{2}} \int_{0}^{+\infty} y^{2 s} \lambda^{s} K_{s}\left(y \lambda^{1 / 2}\right)^{2} d y \\
& =-\frac{\pi^{2} \Gamma(4 s)}{2^{8 s} s^{2} \Gamma(s)^{4}} \lambda^{-1 / 2}
\end{aligned}
$$

Similarly

$$
\int_{0}^{+\infty} G(+\infty, y) \mathcal{K}(y, \lambda)^{2} d y=\frac{1}{2 s} \frac{2^{2-2 s}}{\Gamma(s)^{2}} \int_{0}^{+\infty} y^{2 s+1} \lambda^{s} K_{s}\left(y \lambda^{1 / 2}\right)^{2} d y=\frac{s}{2 s+1} \lambda^{-1} .
$$

Using the Bessel process as a vertical diffusion, one deduces therefore:

Proposition 4.10. Let $G$ be a Lie group of compact type endowed with a biinvariant Riemannian structure. Let $1 \leq i, j \leq n$. Then for every $s \in(0,1)$

$$
\begin{gathered}
\left\|(-L)^{-1 / 2} \mathfrak{X}_{i} f\right\|_{p} \leq \frac{2^{8 s} s^{2} \Gamma(s)^{4}}{4 \pi^{2} \Gamma(4 s)} \cot \left(\frac{\pi}{2 p^{*}}\right)\|f\|_{p}, \\
\left\|\frac{1}{2}\left(\mathfrak{X}_{i} \mathfrak{X}_{j}+\mathfrak{X}_{j} \mathfrak{X}_{i}\right)(-L)^{-1} f\right\|_{p} \leq \frac{2 s+1}{4 s}\left(p^{*}-1\right)\|f\|_{p} .
\end{gathered}
$$


Proof. Since as before the martingale representations for $T_{i} f$ and $S_{i j} f$ give that $\left\|T_{i} f\right\|_{p} \leq \frac{1}{4} \cot \left(\frac{\pi}{2 p^{*}}\right)\|f\|_{p}$ and $\left\|S_{i j} f\right\|_{p} \leq \frac{1}{4}\left(p^{*}-1\right)\|f\|_{p}$, both inequalities follow immediately.

Of course the constant $\frac{2 s+1}{4 s}$ is best for $s \rightarrow 1$ which corresponds to 0-dimensional Bessel process as a vertical diffusion. On the other hand the constant $\frac{2^{8 s} s^{2} \Gamma(s)^{4}}{4 \pi^{2} \Gamma(4 s)}$ is optimal for $s=1 / 2$ which corresponds to 1-dimensional Bessel process (=Brownian motion) as a vertical diffusion.

4.4. Generalized Riesz transform on vector bundles. We consider the framework introduced in Section 3.1 of [8]. Let $M$ be a $n$-dimensional smooth complete Riemannian manifold and let $\mathcal{E}$ be a finite-dimensional vector bundle over $M$. We denote by $\Gamma(M, \mathcal{E})$ the space of smooth sections of this bundle. Let $\nabla$ denote a metric connection on $\mathcal{E}$. We consider an operator on $\Gamma(M, \mathcal{E})$ that can be written as

$$
\mathcal{L}=\mathcal{F}+\nabla_{0}+\sum_{i=1}^{n} \nabla_{i}^{2}
$$

where

$$
\nabla_{i}=\nabla_{\mathfrak{X}_{i}}, \quad 0 \leq i \leq n,
$$

and the $\mathfrak{X}_{i}$ 's are smooth vector fields on $M$ and $\mathcal{F}$ is a smooth symmetric and non positive potential (that is a smooth section of the bundle $\operatorname{End}(\mathcal{E})$ ). We assume that $\mathcal{L}$ is locally subelliptic, non-positive and essentially self-adjoint on the space $\Gamma_{0}(M, \mathcal{E})$ of smooth and compactly supported sections. We consider then a first order differential operator $d_{a}$ on $\Gamma(M, \mathcal{E})$ that can be written as

$$
d_{a}=\sum_{i=1}^{n} a_{i} \nabla_{\mathfrak{X}_{i}}
$$

where $a_{1}, \cdots, a_{n}$ are smooth sections of the bundle $\operatorname{End}(\mathcal{E})$. Assume that $d_{a}$ commutes with $\mathcal{L}$, i.e.

$$
d_{a} \mathcal{L} \eta=\mathcal{L} d_{a} \eta, \quad \eta \in \Gamma(M, \mathcal{E}),
$$

and that

$$
\left\|d_{a} \eta\right\|^{2} \leq C \sum_{i=1}^{n}\left\|\nabla_{\mathfrak{X}_{i}} \eta\right\|^{2}, \quad \eta \in \Gamma(M, \mathcal{E}),
$$

for some constant $C \geq 0$.

The following theorem can then be proved by combining the techniques of this paper with the analysis performed in Section 3.1 of [8].

Theorem 4.11. Let $\Phi:(0,+\infty) \rightarrow \mathbb{C}$ be a complex Borel function. If there exists a finite complex Borel measure $\alpha$ on $\mathbb{R}_{\geq 0}$ such that for every $x \in(0,+\infty)$,

$$
\Phi(x)=\int_{0}^{+\infty} \frac{d \alpha(m)}{\sqrt{x+m}},
$$

then, for every $p>1$ and $\eta \in \Gamma_{0}(M, \mathcal{E})$

$$
\left\|\Phi(-\mathcal{L}) d_{a} \eta\right\|_{p} \leq 6 C\left(p^{*}-1\right)|\alpha|\left(\mathbb{R}_{\geq 0}\right)\|\eta\|_{p} .
$$

Theorem 1.3 follows then from the previous theorem as in Section 3.2 of [8]. 
Acknowledgements. We are grateful to the anonymous referee for the very careful reading and the many comments, including corrections which greatly improved the paper.

\section{REFERENCES}

1. Milton Abramowitz and Irene A. Stegun, Handbook of mathematical functions with formulas, graphs, and mathematical tables, National Bureau of Standards Applied Mathematics Series, vol. 55, For sale by the Superintendent of Documents, U.S. Government Printing Office, Washington, D.C., 1964. MR 016764216

2. Joyce Assaad and El Maati Ouhabaz, Riesz transforms of Schrödinger operators on manifolds, J. Geom. Anal. 22 (2012), no. 4, 1108-1136. MR 29653642

3. Kari Astala, Tadeusz Iwaniec, and Gaven Martin, Elliptic partial differential equations and quasiconformal mappings in the plane, Princeton Mathematical Series, vol. 48, Princeton University Press, Princeton, NJ, 2009. MR 247287523

4. Pascal Auscher, Thierry Coulhon, Xuan Thinh Duong, and Steve Hofmann, Riesz transform on manifolds and heat kernel regularity, Ann. Sci. École Norm. Sup. (4) 37 (2004), no. 6, 911-957. MR 21192421

5. R. Bañuelos and P. J. Méndez-Hernández, Space-time Brownian motion and the BeurlingAhlfors transform, Indiana Univ. Math. J. 52 (2003), no. 4, 981-990. MR 2001941 2, 4, 22, 23

6. Rodrigo Bañuelos, The foundational inequalities of D. L. Burkholder and some of their ramifications, Illinois J. Math. 54 (2010), no. 3, 789-868 (2012). MR 2928339 2, 23

7. Rodrigo Bañuelos and Fabrice Baudoin, Martingale transforms and their projection operators on manifolds, Potential Anal. 38 (2013), no. 4, 1071-1089. MR 30426952

8. Rodrigo Bañuelos, Fabrice Baudoin, and Li Chen, Gundy-Varopoulos martingale transforms and their projection operators on manifolds and vector bundles, Math. Ann. 378 (2020), no. 1-2, 359-388. MR 4150921 1, 2, 4, 13, 25

9. Rodrigo Bañuelos and Krzysztof Bogdan, Lévy processes and Fourier multipliers, J. Funct. Anal. 250 (2007), no. 1, 197-213. MR 23459122

10. Rodrigo Bañuelos and Prabhu Janakiraman, $L^{p}$-bounds for the Beurling-Ahlfors transform, Trans. Amer. Math. Soc. 360 (2008), no. 7, 3603-3612. MR 238623823

11. Rodrigo Bañuelos and Adam Osȩkowski, Martingales and sharp bounds for Fourier multipliers, Ann. Acad. Sci. Fenn. Math. 37 (2012), no. 1, 251-263. MR 292043822

12. - Sharp martingale inequalities and applications to Riesz transforms on manifolds, Lie groups and Gauss space, J. Funct. Anal. 269 (2015), no. 6, 1652-1713. MR 3373431 2, 10

13. Rodrigo Bañuelos and Gang Wang, Sharp inequalities for martingales with applications to the Beurling-Ahlfors and Riesz transforms, Duke Math. J. 80 (1995), no. 3, 575-600. MR 1370109 $2,4,10,23$

14. Dominique Bakry, Étude des transformations de Riesz dans les variétés riemanniennes à courbure de Ricci minorée, Séminaire de Probabilités, XXI, Lecture Notes in Math., vol. 1247, Springer, Berlin, 1987, pp. 137-172. MR 9419801

15. Dominique Bakry and Michel Émery, Diffusions hypercontractives, Séminaire de probabilités, XIX, 1983/84, Lecture Notes in Math., vol. 1123, Springer, Berlin, 1985, pp. 177-206. MR 8894769

16. Dominique Bakry, Ivan Gentil, and Michel Ledoux, Analysis and geometry of Markov diffusion operators, Grundlehren der Mathematischen Wissenschaften [Fundamental Principles of Mathematical Sciences], vol. 348, Springer, Cham, 2014. MR 315520915

17. Fabrice Baudoin, Diffusion processes and stochastic calculus, EMS Textbooks in Mathematics, European Mathematical Society (EMS), Zürich, 2014. MR 3236242 5, 17

18. Fabrice Baudoin, Geometric inequalities on riemannian and sub-riemannian manifolds by heat semigroups techniques, arXiv:1801.05702, to appear in Levico Summer School Lecture notes. (2018). 4, 5, 8, 16

19. Andrei N. Borodin and Paavo Salminen, Handbook of Brownian motion-facts and formulae, second ed., Probability and its Applications, Birkhäuser Verlag, Basel, 2002. MR 1912205 6, $8,14,15$

20. Luis Caffarelli and Luis Silvestre, An extension problem related to the fractional Laplacian, Comm. Partial Differential Equations 32 (2007), no. 7-9, 1245-1260. MR 23544932 
21. K. P. Choi, A sharp inequality for martingale transforms and the unconditional basis constant of a monotone basis in $L^{p}(0,1)$, Trans. Amer. Math. Soc. 330 (1992), no. 2, 509-529. MR 103466122

22. Xuan Thinh Duong, Adam Sikora, and Lixin Yan, Weighted norm inequalities, Gaussian bounds and sharp spectral multipliers, J. Funct. Anal. 260 (2011), no. 4, 1106-1131. MR 274701711

23. Sylvestre Gallot, Dominique Hulin, and Jacques Lafontaine, Riemannian geometry, third ed., Universitext, Springer-Verlag, Berlin, 2004. MR 20880275

24. Stefan Geiss, Stephen Montgomery-Smith, and Eero Saksman, On singular integral and martingale transforms, Trans. Amer. Math. Soc. 362 (2010), no. 2, 553-575. MR 2551497 4, 22, 23

25. Loukas Grafakos, Classical Fourier analysis, third ed., Graduate Texts in Mathematics, vol. 249, Springer, New York, 2014. MR 324373410

26. Loukas Grafakos and Lenka Slavíková, A sharp version of the Hörmander multiplier theorem, Int. Math. Res. Not. IMRN (2019), no. 15, 4764-4783. MR 398866811

27. A. A. Grigoryan, Stochastically complete manifolds, Dokl. Akad. Nauk SSSR 290 (1986), no. 3, 534-537. MR 8603245

28. Richard F. Gundy and Nicolas Th. Varopoulos, Les transformations de Riesz et les intégrales stochastiques, C. R. Acad. Sci. Paris Sér. A-B 289 (1979), no. 1, A13-A16. MR 5456712

29. I. I. Hirschman and D. V. Widder, The convolution transform, Princeton University Press, Princeton, N. J., 1955. MR 00737463

30. Lars Hörmander, Hypoelliptic second order differential equations, Acta Math. 119 (1967), 147-171. MR 22247416

31. Tadeusz Iwaniec and Gaven Martin, Riesz transforms and related singular integrals, J. Reine Angew. Math. 473 (1996), 25-57. MR 1390681 4, 23

32. David Jerison and Antonio Sánchez-Calle, Subelliptic, second order differential operators, Complex analysis, III (College Park, Md., 1985-86), Lecture Notes in Math., vol. 1277, Springer, Berlin, 1987, pp. 46-77. MR 9223344

33. E. Lenglart, D. Lépingle, and M. Pratelli, Présentation unifiée de certaines inégalités de la théorie des martingales, Seminar on Probability, XIV (Paris, 1978/1979) (French), Lecture Notes in Math., vol. 784, Springer, Berlin, 1980, With an appendix by Lenglart, pp. 26-52. MR 58010714

34. Terry R. McConnell, On Fourier multiplier transformations of Banach-valued functions, Trans. Amer. Math. Soc. 285 (1984), no. 2, 739-757. MR 75250111

35. S. A. Molčanov and E. Ostrovskiŭ, Symmetric stable processes as traces of degenerate diffusion processes, Teor. Verojatnost. i Primenen. 14 (1969), 127-130. MR 02476682

36. Giampiero Palatucci and Tuomo Kuusi (eds.), Recent developments in nonlocal theory, De Gruyter, Berlin, 2018. MR 38242062

37. Jim Pitman and Marc Yor, Bessel processes and infinitely divisible laws, Stochastic integrals (Proc. Sympos., Univ. Durham, Durham, 1980), Lecture Notes in Math., vol. 851, Springer, Berlin, 1981, pp. 285-370. MR 62099516

38. Daniel Revuz and Marc Yor, Continuous martingales and Brownian motion, third ed., Grundlehren der Mathematischen Wissenschaften [Fundamental Principles of Mathematical Sciences], vol. 293, Springer-Verlag, Berlin, 1999. MR 1725357 6, 9

39. Zhong Wei Shen, $L^{p}$ estimates for Schrödinger operators with certain potentials, Ann. Inst. Fourier (Grenoble) 45 (1995), no. 2, 513-546. MR 13435602

40. Pablo Raúl Stinga and José Luis Torrea, Extension problem and Harnack's inequality for some fractional operators, Comm. Partial Differential Equations 35 (2010), no. 11, 20922122. MR 27540802,8

41. Karl-Theodor Sturm, Analysis on local Dirichlet spaces. I. Recurrence, conservativeness and $L^{p}$-Liouville properties, J. Reine Angew. Math. 456 (1994), 173-196. MR 13014565

42. A. Volberg and F. Nazarov, Heat extension of the Beurling operator and estimates for its norm, Algebra i Analiz 15 (2003), no. 4, 142-158. MR 2068982 22, 23

43. Shinzo Watanabe, On time inversion of one-dimensional diffusion processes, Z. Wahrscheinlichkeitstheorie und Verw. Gebiete 31 (1974/75), 115-124. MR 36573116

44. D. V. Widder, The Stieltjes transform, Trans. Amer. Math. Soc. 43 (1938), no. 1, 7-60. MR 15019333 
Department of Mathematics, Purdue University, West Lafayette, IN 47907

Email address: banuelos@math.purdue.edu

Department of Mathematics, University of Connecticut, Storrs, CT 06269

Email address: fabrice.baudoin@uconn.edu

Department of Mathematics, Louisiana State University, Baton Rouge, LA 70803

Email address: lichen@math.lsu.edu

Department of Mathematics, Johns Hopkins University, Baltimore, MD 21218

Email address: sire@math.jhu.edu 